\title{
Review \\ On Markov Moment Problem, Polynomial Approximation on Unbounded Subsets, and Mazur-Orlicz Theorem
}

\author{
Octav Olteanu (D)
}

Citation: Olteanu, O. On Markov Moment Problem, Polynomial Approximation on Unbounded Subsets, and Mazur-Orlicz Theorem. Symmetry 2021, 13, 1967. https:// doi.org/10.3390/sym13101967

Academic Editors: Vyacheslav Yukalov, Igor Andrianov and Simon L. Gluzman

Received: 3 October 2021

Accepted: 15 October 2021

Published: 18 October 2021

Publisher's Note: MDPI stays neutral with regard to jurisdictional claims in published maps and institutional affiliations.
Department Mathematics-Informatics, University Politehnica of Bucharest, Splaiul Independenţei 313, 060042 Bucharest, Romania; octav.olteanu50@gmail.com

\begin{abstract}
We review earlier and recent results on the Markov moment problem and related polynomial approximation on unbounded subsets. Such results allow proving the existence and uniqueness of the solutions for some Markov moment problems. This is the first aim of the paper. Our solutions have a codomain space a commutative algebra of (linear) symmetric operators acting from the entire real or complex Hilbert space $H$ to $H$; this algebra of operators is also an order complete Banach lattice. In particular, Hahn-Banach type theorems for the extension of linear operators having a codomain such a space can be applied. The truncated moment problem is briefly discussed by means of reference citations. This is the second purpose of the paper. In the end, a general extension theorem for linear operators with two constraints is recalled and applied to concrete spaces. Here polynomial approximation plays no role. This is the third aim of this work.
\end{abstract}

Keywords: extension of linear operators; polynomial approximation; Markov moment problem; existence of a solution; uniqueness of the solution; quadratic forms; moment determinate measure; symmetric operators; Mazur-Orlicz theorem

\section{Introduction}

Originally, the moment problem was formulated by T. Stieltjes in 1894-1895 (see [1]): find the repartition of the positive mass on the nonnegative semiaxis, if the moments of arbitrary orders $k(k=0,1,2, \ldots)$ are given. Specifically, in the Stieltjes moment problem, a sequence of real numbers $\left(y_{k}\right)_{k \geq 0}$ is given, and one looks for a nondecreasing real function $\sigma(t)(t \geq 0)$, which verifies the moment conditions: $\int_{0}^{\infty} t^{k} d \sigma=y_{k},(k=0,1,2, \ldots)$. If such a function $\sigma$ does exist, the sequence $\left(y_{k}\right)_{k \geq 0}$ is called a Stieltjes moment sequence. A Hamburger moment sequence is a sequence $\left(y_{k}\right)_{k \geq 0}$ for which there exists a positive regular Borel measure $\mu$ on $\mathbb{R}$, such that $\int_{\mathbb{R}} t^{k} d \mu=y_{k}, k=0,1, \ldots$ The existence, uniqueness, and eventually the construction of the solution $d \sigma$ starting from its moments $\int_{0}^{\infty} t^{k} d \sigma, k \in \mathbb{N}$ is under attention. The problems stated above have been generalized as follows: being given a sequence $\left(y_{j}\right)_{j \in \mathbb{N}^{n}}$ of real numbers and a closed subset $F \subseteq \mathbb{R}^{n}, n \in\{1,2, \ldots\}$, find a positive regular Borel measure $\mu$ on $F$ such that $\int_{F} t^{j} d \mu=y_{j}, j \in \mathbb{N}^{n}$. This is the full moment problem. The existence, uniqueness, and construction of the unknown solution $\mu$ are the focus of attention. The numbers $y_{j}, j \in \mathbb{N}^{n}$ are called the moments of the measure $\mu$. When a sandwich condition on the solution is required, we have a Markov moment problem. The moment problem is an inverse problem since the measure $\mu$ is not known. It must be "found", starting from its moments. The direct problem could be: being given the measure $\mu$, find its moments.

We use the following notations:

$$
\begin{gathered}
\mathbb{N}=\{0,1,2, \ldots\}, \mathbb{R}_{+}=[0, \infty], \\
\varphi_{j}(t)=t^{j}=t_{1}^{j_{1}} \ldots t_{n}^{j_{n}}, j=\left(j_{1}, \ldots, j_{n}\right) \in \mathbb{N}^{n}, t=\left(t_{1}, \ldots, t_{n}\right) \in F, n \in \mathbb{N}, n \geq 1 .
\end{gathered}
$$


$\mathcal{P}=\mathbb{R}\left[t_{1}, \ldots, t_{n}\right]$ will be the vector space of all polynomials with real coefficients, and $\mathcal{P}_{+}=\mathcal{P}_{+}(F)$ denotes the convex cone of all polynomials $p \in \mathcal{P}$ which satisfy the condition $p(t) \geq 0$ for all $t \in F$. If $F$ is closed and unbounded, then we denote by $C_{0}(F)$ the vector space of all the real valued continuous compactly supported functions defined on $F$. If $y_{j}, j \in \mathbb{N}^{n}$ are elements of a Banach lattice $Y$, a solution for the moment problem is a positive linear operator $T: X \rightarrow Y$, satisfying the moment interpolation conditions

$$
T\left(\varphi_{j}\right)=y_{j}, j \in \mathbb{N}^{n},
$$

where $X$ is a Banach lattice containing $\mathcal{P}$ and $C_{0}(F)$. An important particular case is when $X=L_{v}^{1}(F)$, where $v$ is a positive regular Borel moment determinate measure on $F$, with finite moments of all orders. Recall that $v$ is called a moment determinate measure if it is uniquely determined by its moments $\int_{F} t^{j} d v, j \in \mathbb{N}^{n}$ (or, equivalently, by its values on $\mathcal{P}$ ). $Y$ may be a commutative Banach algebra of self-adjoint operators acting on a Hilbert space, which is also an order complete Banach lattice. In this case, we have an operator valued moment problem. If we define the linear operator

$$
T_{0}: \mathcal{P} \rightarrow Y, T_{0}\left(\sum_{j \in J_{0}} \alpha_{j} \varphi_{j}\right):=\sum_{j \in J_{0}} \alpha_{j} y_{j},
$$

assuming that $\mathrm{T}_{0}$ satisfies the positivity condition:

$$
p \in \mathcal{P}, p(t) \geq 0 \forall t \in F \Rightarrow T_{0}(p) \geq \mathbf{0},
$$

then the existence of a (positive) solution $T$ for the moment problem defined by (1) is equivalent to the existence of a positive linear extension $T$ of $T_{0}$ from $\mathcal{P}$ to the entire Banach lattice $X$.

For basic notions and terminology used in this paper see the monographs [1-7]. In [8], the main extension result (Kantorovich theorem) on the extension of positive linear operators is proved. It will be applied in Section 3 below. If $Y=\mathbb{R}$ and (3) is verified, the Haviland theorem [9] ensures the existence of a positive regular Borel measure $\mu$ on $F$, such that

$$
\int_{F} t^{j} d \mu=T_{0}\left(\varphi_{j}\right)=y_{j}, j \in \mathbb{N}^{n} .
$$

If these equalities hold true, then we say that $\left(y_{j}\right)_{j \in \mathbb{N}^{n}}$ is a moment sequence (or a sequence of moments) on $F$, and $\mu$ is a representing measure for $T_{0}$ defined by (2). In this paper, by a measure on $F$ we mean a positive regular Borel measure having finite moments of all orders on F. Moments appear in physics, probabilities, and statistics, as discussed in the Introduction of [3]. The papers [10-21] refer to various aspects of the moment problem or contain related results on polynomial functions. In ref. [22], extension and controlled regularity of linear operators is applied to characterize the monotone increasing convex operators on a convex cone. The fact that any positive linear operator acting between two ordered Banach spaces is continuous is also proved. The papers [23-29] are devoted to some main aspects of the Markov moment problem or to the extension of linear operators with two constraints (the extension satisfies a sandwich condition). In the paper [29], such general theorems are applied to the Markov moment problem and Mazur-Orlicz type theorems for operators, without using polynomial approximation. On the other hand, polynomial approximations are reviewed and applied to the existence and uniqueness of the solution for some full Markov moment problems. The main difference between the onedimensional and the multidimensional cases of the moment problem, in terms of a sequence of numbers $\left(y_{j}\right)_{j \in \mathbb{N}^{n}}$, can be formulated in the following way: for any $n \in\{1,2 \ldots\}$, any moment sequence $\left(y_{j}\right)_{j \in \mathbb{N} n}$ is positive semi-definite; that is: 


$$
\sum_{i, j \in J_{0}} \lambda_{i} \lambda_{j} y_{i+j} \geq 0
$$

for any finite subset $J_{0} \subset \mathbb{N}^{n}$ and any $\left\{\lambda_{j} ; j \in J_{0}\right\} \subset \mathbb{R}$. Indeed,

$$
\sum_{i, j \in J_{0}} \lambda_{i} \lambda_{j} y_{i+j}=\sum_{i, j \in J_{0}} \lambda_{i} \lambda_{j} \int_{F} t^{i+j} d \mu=\int_{F}\left(\sum_{j \in J_{0}} \lambda_{j} t^{j}\right)^{2} d \mu \geq 0,
$$

For $n=1$, the converse is true, since any non-negative polynomial on $\mathbb{R}$ is a sum of (two) squares of polynomials, and a square of a polynomial can be written as $\left(\sum_{j \in J_{0}} \lambda_{j} \varphi_{j}\right)^{2}=$ $\sum_{i, j \in J_{0}} \lambda_{i} \lambda_{j} \varphi_{i+j}$; then, one applies Haviland's theorem. Thus, for $n=1$ a sequence of real numbers is a moment sequence if, and only if, it is positive semi-definite. For $n \geq 2$, there exist positive semi-definite sequences that are not moment sequences (see [2]). On $\mathbb{R}^{n}, n \geq 2$, there exist nonnegative polynomials which are not sums of squares (see $[3,10,12])$. The first example of such a polynomial was discussed in [12]. The rest of the paper is organized as follows: Section 2 briefly summarizes the methods used in this work. In Section 3, the main results are stated, and some of them are also proved or discussed. Section 4 discusses the results mentioned above and concludes the paper.

\section{Methods}

The methods applied in the paper are partially motivated by the importance of solving the old and modern aspects of the moment and related problems. Here are the main methods used in the sequel:

(1) Extension of positive linear operators (see [8] for the operator version). Extension of linear operators, satisfying a sandwich condition (see [25]). Such results are used in the existence of a solution for some Markov moment problems and the Mazur-Orlicz theorem (see [29]).

(2) Elements of determinacy of measures on $\mathbb{R}$ and on $\mathbb{R}_{+}$(the one-dimensional case) (see [3] and mainly [13] for checkable sufficient conditions on determinacy).

(3) Polynomial approximation of nonnegative continuous compactly supported functions defined on a closed unbounded subset $F \subseteq \mathbb{R}^{n}$ by dominating polynomials. The approximation holds in $L_{v}^{1}(F)$, where $v$ is a moment determinate measure on $F$. If $F=\mathbb{R}^{n}, v=v_{1} \times \cdots \times v_{n}$, and $v_{j}$ is moment determinate measure on $\mathbb{R}, j=1, \ldots, n$, the approximation mentioned above holds by means of finite sums of polynomials $p_{1} \otimes \cdots \times \otimes p_{n}$, where $p_{j}$ is a nonnegative polynomial on $\mathbb{R}, j=1, \ldots, n$ (see formula (4) below for the notation $p_{1} \otimes \cdots \times \otimes p_{n}$ ). Since each $p_{j}$ is the sum of (two) squares of polynomials in $\mathbb{R}[t]$, we know the expression of such approximating polynomials in terms of sums of squares. A similar method works when we replace $\mathbb{R}^{n}$ by $\mathbb{R}_{+}^{n}$ $\left(p \in \mathcal{P}_{+}\left(\mathbb{R}_{+}\right) \Leftrightarrow p(t)=p_{1}^{2}(t)+t p_{2}^{2}(t), t \in \mathbb{R}_{+}\right.$, for some $\left.p_{1}, p_{2} \in \mathbb{R}[t]\right)$. These results lead to the characterization of the existence and uniqueness of the solutions for the multidimensional Markov moment problems in terms of quadratic forms. Moreover, the positivity of some linear continuous operators in terms of quadratic forms is obtained as well (see [27]).

(4) Results, comments, and remarks on the truncated moment problem are mentioned in Section 3.3 (see $[20,21,23,24,28]$ ).

\section{Results}

\subsection{On Determinacy: The One-Dimensional Case}

In what follows, we review some known aspects of the problem of determinacy of a measure, in the one-dimensional case. A Hamburger moment sequence is determinate if it has a unique representing measure, while a Stieltjes moment sequence is called determinate if it has only one representing measure supported on $[0, \infty]$. The Carleman theorem (the next result) contains a powerful sufficient condition for determinacy. 
Theorem 1 (Carleman condition; see [3], Theorem 4.3). Suppose that $\boldsymbol{y}=\left(y_{n}\right)_{n \in \mathbb{N}}$ is a positive semi-definite sequence $\left(\sum_{i, j=0}^{n} y_{i+j} \lambda_{i} \lambda_{j} \geq 0\right.$ for all $n \in \mathbb{N}$ and arbitrary $\left.\lambda_{j} \in \mathbb{R}, j=0, \ldots, n\right)$.

(i) If $y$ satisfies the Carleman condition

$$
\sum_{n=1}^{\infty} y_{2 n}^{-\frac{1}{2 n}}=+\infty
$$

then $y$ is a determinate Hamburger moment sequence.

(ii) If in addition $\left(y_{n+1}\right)_{n \in \mathbb{N}}$ is positive semi-definite and

$$
\sum_{n=1}^{\infty} y_{n}^{-\frac{1}{2 n}}=+\infty
$$

then $y$ is a determinate Stieltjes moment sequence.

The following theorem of Krein consists of a sufficient condition for indeterminacy (for measures given by densities).

Theorem 2 (Krein condition; see [3], Theorem 4.14). Let $f$ be a nonnegative Borel function on $\mathbb{R}$. Suppose that the measure $\mu$ defined by $d \mu=f(t) d t$ is a Radon measure on $\mathbb{R}$ and has finite moments $y_{n}:=\int_{\mathbb{R}} t^{n} d \mu$ for all $n \in \mathbb{N}$. If

$$
\int_{\mathbb{R}} \frac{-\ln (f(x))}{1+x^{2}} d x<+\infty,
$$

then the moment sequence $y=\left(y_{n}\right)_{n \in \mathbb{N} \text {. }}$ is $M-$ indeterminate.

Next, we give new checkable sufficient conditions on distributions of random variables that imply the Carleman condition, ensuring determinacy. Consider two random variables $V \sim \Psi, V$ with values in $\mathbb{R}, W \sim \Phi, W$ with values in $\mathbb{R}_{+}$. Assume that both $\Psi$ and $\Phi$ have continuous derivatives and let $f=\Psi^{\prime}$ and $g=\Phi^{\prime}$, respectively, be the corresponding densities. All moments of $\Psi, \Phi$ are assumed to be finite. The symbol $\nearrow$ used below has the usual meaning of "monotone increasing".

Theorem 3 (see [16], Theorem 1; Hamburger case). Assume that the density f of $\Psi$ is symmetric on $\mathbb{R}$ and continuous and strictly positive outside an interval $\left[-t_{0}, t_{0}\right], t_{0}>1$, such that the following conditions hold:

$$
\int_{|t| \geq t_{0}} \frac{-\ln f(t)}{t^{2} \ln (|t|)} d t=\infty,
$$

$\frac{-\ln f(t)}{\ln t} \nearrow \infty$ as $t_{0} \leq t \rightarrow \infty$

Then $V \sim \Psi$ satisfies Carleman's condition, and hence it is $M$-determinate.

Theorem 4 (see [13], Theorem 2; Stieltjes case). Assume that the density $g$ of $\Phi$ is continuous and strictly positive on $[a, \infty]$ for some $a>1$ such that the following conditions hold:

$$
\int_{a}^{\infty} \frac{-\ln g\left(t^{2}\right)}{t^{2} \ln t} d t=\infty,
$$

$\frac{-\ln g(t)}{\ln t} \nearrow \infty$ as $a \leq t \rightarrow \infty$.

Under these conditions, $W \sim \Phi$ satisfies Carleman's condition, and hence it is $M$-determinate.

Example 1. The distribution function $\Phi$ having a density $g(u)=\exp (-u), u \in \mathbb{R}_{+}$, satisfies the conditions of Theorem 4, hence it is M-determinate. 
Remark 1. The problem of determinacy of measures on $\mathbb{R}^{n}, \mathbb{R}_{+}^{n}, n \geq 2$, is much more difficult than that for the case $n=1$. In the next subsection, we partially solve this problem (see Lemmas 3-5, Theorem 8, as well as their corollaries stated or proved in Section 3.2).

\subsection{Polynomial Approximation and Some of Its Applications}

As we have seen in Section 1, characterizing moment sequences in terms of the existence of a representing measure on $\mathbb{R}^{n}, n \geq 2$ is not as simple as in one dimension. However, important results have been proved for compact subsets with nonempty interiors in $\mathbb{R}^{n}$ (see [14]) and for semi-algebraic compact subsets in $\mathbb{R}^{n}$, for arbitrary $n \geq 2, n \in \mathbb{N}$ (see [3,15-17]). For all such compact subsets involved in the papers [14-17], the existence of a representing measure for a given sequence of numbers has been characterized by pointing out special nonnegative polynomials on the compact under attention. Moreover, for any polynomial taking (strictly) positive values at all points of the compact subset, decomposition theorems were proved. Namely, the explicit form of a positive polynomial as the sum of special polynomials obtained by the aid of those polynomials that define the compact subset has been found. In the case of the semi-algebraic compact subset, this last result is named Schmüdgen's Positivstellensatz. Its statement involves not only the polynomials that define the semi-algebraic compact but also sums of squares of other polynomials. There also exists Putinar's Positivstellensatz. They were continued and generalized in [17], where the case of unbounded semi-algebraic sets is studied, using deep results in operator theory. To solve Markov moment problems on $\mathbb{R}^{n}, \mathbb{R}_{+}^{n}$, where the explicit form of nonnegative polynomials cannot be expressed in terms of sums of squares, we use the approximation of any nonnegative continuous compactly supported function $\psi$ by sums of special polynomials of the form

$$
\left(p_{1} \otimes \cdots \otimes p_{n}\right)\left(t_{1}, \ldots, t_{n}\right)=p_{1}\left(t_{1}\right) \cdots p_{n}\left(t_{n}\right)
$$

where each $p_{j}$ is nonnegative on $\mathbb{R}\left(\right.$ or on $\left.\mathbb{R}_{+}\right), j=1, \ldots, n$. We recall that

$$
\begin{gathered}
p(t) \geq 0 \forall t \in \mathbb{R} \Leftrightarrow p(t)=p_{1}^{2}(t)+p_{2}^{2}(t), t \in \mathbb{R} \\
p(t) \geq 0 \forall t \in \mathbb{R}_{+} \Leftrightarrow p(t)=p_{1}^{2}(t)+t p_{2}^{2}(t), t \in \mathbb{R}_{+}
\end{gathered}
$$

for some $p_{1}, p_{2} \in \mathbb{R}[t]$. Thus we can express approximating polynomials in terms of sums of squares. This leads to characterizations for the existence and uniqueness of the full Markov moment problem in terms of quadratic forms. The sums of special nonnegative polynomials (4) approximate $\psi$ and dominate $\psi$. The approximation holds in the space $L_{v}^{1}\left(\mathbb{R}^{n}\right)$, (respectively in $L_{v}^{1}\left(\mathbb{R}_{+}^{n}\right)$ ), where $v=v_{1} \times \cdots \times v_{n}$, with each $v_{j}$ being a moment determinate measure on $\mathbb{R}$ (respectively on $\mathbb{R}_{+}$), with finite moments of all orders. We start with polynomial approximation by nonnegative polynomials on $\mathbb{R}_{+}$, for continuous nonnegative functions. The proof of the next results is based on the Stone-Weierstrass theorem and the properties of partial sums of the alternate Leibniz series obtained from the Taylor series expansion of the functions $e_{k}(t)=\exp (-k t), t \in[0, \infty], k \in \mathbb{N}$.

First, we recall the Kantorovich extension result for positive linear operators [8]. Let $X_{1}$ be an ordered vector space whose positive cone $X_{1,+}$ generates $X_{1}\left(X_{1}=X_{1,+}-X_{1,+}\right)$. Recall that in such an ordered vector space $X_{1}$, a vector subspace $S$ is called a majorizing subspace if for any $x \in X_{1}$ there exists $s \in S$ such that $x \leq s$. The following Kantorovich theorem on the extension of positive linear operators holds true:

Theorem 5 (see [8], Theorem 1.2.1). Let $X_{1}$ be an ordered vector space whose positive cone generates $X_{1}, X_{0} \subset X_{1}-a$ majorizing vector subspace, $Y$ an order complete vector space, and $T_{0}: X_{0} \rightarrow Y$ a positive linear operator. Then, $T_{0}$ admits a positive linear extension $T: X_{1} \rightarrow Y$.

Another extension type result for linear operators, satisfying a sandwich condition, formulated in terms of the Markov moment problem is stated as follows: 
Theorem 6 (see [25], Theorem 4). Let X be an ordered vector space, $Y$ an order complete vector lattice, $\left\{x_{j}\right\}_{j \in J},\left\{y_{j}\right\}_{j \in J}$ families of elements in $X$ and $Y$, respectively, and $T_{1}, T_{2} \in L(X, Y)$ two linear operators. The following statements are equivalent:

(a) There is a linear operator $T \in L(X, Y)$, such that

$$
T_{1}(x) \leq T(x) \leq T_{2}(x), x \in X_{+}, T\left(x_{j}\right)=y_{j}, j \in J
$$

(b) For any finite subset $J_{0} \subset J$, and any $\left\{\alpha_{j}\right\}_{j \in J_{0}} \subset \mathbb{R}$, the following implication holds true:

$$
\left(\sum_{j \in J_{0}} \alpha_{j} x_{j}=\psi_{2}-\psi_{1}, \quad \psi_{1}, \psi_{2} \in X_{+}\right) \Rightarrow \sum_{j \in J_{0}} \alpha_{j} y_{j} \leq T_{2}\left(\psi_{2}\right)-T_{1}\left(\psi_{1}\right)
$$

(c) If $X$ is a vector lattice, then assertions ( $a)$ and (b) are equivalent to (c), where (c) $T_{1}(w) \leq$ $T_{2}(w)$ for all $w \in X_{+}$and for any finite subset $J_{0} \subset J$ and $\forall\left\{\alpha_{j} ; j \in J_{0}\right\} \subset \mathbb{R}$, we have

$$
\sum_{j \in J_{0}} \alpha_{j} y_{j} \leq T_{2}\left(\left(\sum_{j \in J_{0}} \alpha_{j} x_{j}\right)^{+}\right)-T_{1}\left(\left(\sum_{j \in J_{0}} \alpha_{j} x_{j}\right)^{-}\right)
$$

Theorem 6 can be obtained from a more general result proved in [26].

Lemma 1 (see [28], Lemma 1). Let $\psi: \mathbb{R}_{+}=[0, \infty] \rightarrow \mathbb{R}_{+}$be a continuous function, such that $\lim _{t \rightarrow \infty} \psi(t)$ exists in $\mathbb{R}_{+}$. Then there is a decreasing sequence $\left(h_{l}\right)_{l}$ in $\operatorname{Span}\left\{e_{k} ; k \in \mathbb{N}\right\}$, where the functions $e_{k} ; k \in \mathbb{N}$ are defined as follows:

$$
e_{k}(t)=\exp (-k t), t \in[0, \infty[, k \in \mathbb{N},
$$

such that $h_{l}(t) \geq \psi(t), t \geq 0, l \in \mathbb{N}=\{0,1,2, \ldots\}$, limh $_{l}=\psi$ uniformly on $[0, \infty)$. There exists a sequence of polynomial functions $\left(\tilde{p}_{l}\right)_{l \in \mathbb{N}}, \widetilde{p}_{l} \geq h_{l} \geq \psi$, lim $\tilde{p}_{l}=\psi$, uniformly on compact subsets of $\mathbb{R}_{+}$. In particular, such polynomial approximation holds for nonnegative continuous compactly supported functions $\psi: \mathbb{R}_{+} \rightarrow \mathbb{R}_{+}$.

In applications, the preceding lemma could be useful in order to prove a similar type of result for continuous functions defined only on a compact subset $K \subset \mathbb{R}_{+}$, taking values in $\mathbb{R}_{+}$. For such a function as $\varphi: K \rightarrow \mathbb{R}_{+}$, one denotes by $\varphi_{0}: \mathbb{R}_{+} \rightarrow \mathbb{R}_{+}$the extension of $\varphi$, which satisfies $\varphi_{0}(t)=0$ for all $t \in \mathbb{R}_{+} \backslash K$. From Lemma 1 we infer the next result. To this aim, we observe that $\varphi_{0}$ is dominated by nonnegative continuous compactly supported functions $\psi: \mathbb{R}_{+} \rightarrow \mathbb{R}_{+}, \psi(t)=\varphi_{0}(t)=\varphi(t)$ for all $t \in K$. Then one applies Lemma 1 .

Lemma 2 (see [28], Lemma 2). If $K \subset \mathbb{R}_{+}$is a compact subset, and $\varphi: K \rightarrow \mathbb{R}_{+}$a continuous function, then there exists a sequence $\left(\widetilde{p}_{l}\right)_{l \in \mathbb{N}}$ of polynomial functions, such that $\widetilde{p}_{l} \geq \varphi_{0}$ on $\mathbb{R}_{+},\left.\widetilde{p}_{l}\right|_{K} \rightarrow \varphi, l \rightarrow \infty$, uniformly on $K$.

From Lemma 2 and Theorem 5 (where $X_{1}$ stands for $C(K)$ and $X_{0}$ stands for the subspace $\mathcal{P}$ of all polynomial functions), the next corollary follows easily. We recall a wellknown important example of an order complete Banach lattice $Y$ of self-adjoint operators acting on a complex or real Hilbert space $H$. Let $\mathcal{A}=\mathcal{A}(H)$ be the ordered vector space of all of the self-adjoint operators acting on $H$, and let $V \in \mathcal{A}$. The natural order relation on $\mathcal{A}$ is $U \leq W$ if, and only if:

$$
\langle U h, h\rangle \leq\langle W h, h\rangle \text { for all } h \text { in } H \text {. }
$$


One can prove that $\mathcal{A}$ with this ordering is not a lattice. Therefore, it is interesting to fix $A \in \mathcal{A}$ and define the following:

$\mathrm{Y}_{1}(\mathrm{~A})=\{\mathrm{U} \in \mathcal{A} ; \mathrm{UA}=\mathrm{AU}\}, \mathrm{Y}=\mathrm{Y}(\mathrm{A})=\left\{\mathrm{W} \in \mathrm{Y}_{1}(\mathrm{~A}) ; \mathrm{WU}=\mathrm{UW}, \forall \mathrm{U} \in \mathrm{Y}_{1}(\mathrm{~A})\right\}$

Then, $Y(A)$ is an order complete Banach lattice (and a commutative real algebra), as discussed in [5]. If $U \in \mathcal{A}$, we denote by $\sigma(U)$ the spectrum of $U$, and by $d E_{U}$ the spectral measure attached to $U$. As usual, one denotes $\varphi_{j}(t)=t^{j}, j \in \mathbb{N}, t \in \mathbb{R}_{+}$. We recall that any symmetric linear operator from the entire space $H$ to $H$ is continuous, hence is self-adjoint. This follows quite easily from the closed graph theorem. Since all symmetric operators appearing in what follows are defined on the entire Hilbert space $H$, they are self-adjoint operators. Conversely, by definition, any self-adjoint operator acting on $H$ is symmetric and continuous. Therefore, in this paper, there will be no difference between these two notions. As is well-known, the spectrum of such an operator is a compact subset of the real line. If $H=\mathbb{R}^{n}, n \in \mathbb{N}, n \geq 2$, then $\mathcal{A}$ is isomorphic to the space $\operatorname{Sym}(n, \mathbb{R})$ of all $n \times n$ symmetric matrices with real entries. See [29], p. 11 for an example related to this space.

Corollary 1 (see [28], Corollary 1). With the above notations, assume that $A$ is a positive selfadjoint operator acting on $H, Y=Y(A)$ is the space defined by Equation (5), and $\left(B_{j}\right)_{j \in \mathbb{N}}$ is a sequence of operators in $Y(A)$. The following statements are equivalent:

(a) There exists a unique positive linear operator $T: C(\sigma(A)) \rightarrow Y$, such that

$$
T\left(\varphi_{j}\right)=B_{j}, j \in \mathbb{N}, T(\varphi) \leq \int_{\sigma(A)} \varphi(t) d E_{A}, \varphi \in(C(\sigma(A)))_{+},\|T\| \leq 1
$$

(b) For any polynomial $\sum_{j=0}^{m} \alpha_{j} \varphi_{j} \geq \mathbf{0}$ on $\sigma(A)$, the result is $\sum_{j=0}^{m} \alpha_{j} B_{j} \geq \mathbf{0}$; if $J_{0} \subset \mathbb{N}$ is an arbitrary finite subset, and $\left\{\lambda_{j} ; j \in J_{0}\right\} \subset \mathbb{R}$, then the following inequalities hold:

$$
\sum_{i, j \in J_{0}} \lambda_{i} \lambda_{j} B_{i+j+l} \leq \sum_{i, j \in J_{0}} \lambda_{i} \lambda_{j} A^{i+j+l}, l \in\{0,1 .\}
$$

Proof. $(b) \Rightarrow(a)$. We define $T_{0}: \mathcal{P} \rightarrow Y(A)$ by $T_{0}\left(\sum_{j \in J_{0}} \alpha_{j} \varphi_{j}\right):=\sum_{j \in J_{0}} \alpha_{j} B_{j}$, where $J_{0} \subset \mathbb{N}$ is an arbitrary finite subset, $\alpha_{j} \in \mathbb{R}, j \in J_{0}$. Then, $T_{0}$ is linear and, according to the first condition (b), $T_{0}(p) \geq \mathbf{0}$ for all polynomials $p$ with $p(t) \geq 0 \forall t \in \sigma(A)$. On the other hand, for each $g \in C(\sigma(A))$, there exists a constant function $c$, such that $g(t) \leq c$ for all $t \in \sigma(A)$. According to Theorem 5, $T_{0}$ has a linear positive extension $T: C(\sigma(A)) \rightarrow Y$. Next, we prove that $T$ is continuous (and its norm can be determined). This can be shown for any positive linear operator, in a more general framework. Namely, any positive linear operator acting between two ordered Banach spaces is continuous (see [6] and/or [21]). Here, we are interested only in our problem, when the norm of the involved positive linear operator can be determined. Indeed, for an arbitrary $g \in C(\sigma(A))$, we can write:

$$
\pm T(g)=T( \pm g) \leq T(|g|) \leq T(\|g\| \mathbf{1})=\|g\| T(\mathbf{1}) \Rightarrow|T(g)| \leq\|g\| T(\mathbf{1})
$$

Since the norm on $Y(A)$ is solid $\left(\left|y_{1}\right| \leq\left|y_{2}\right| \Rightarrow y_{1} \leq y_{2}\right)$, the preceding inequality leads to:

$$
\|T(g)\| \leq\|g\|\|T(\mathbf{1})\|, g \in C(\sigma(A)) \Rightarrow\|T\| \leq\|T(\mathbf{1})\|=\left\|T_{0}\left(\varphi_{0}\right)\right\|=\left\|B_{0}\right\| .
$$

On the other hand, clearly $\|T(\mathbf{1})\| \leq\|T\|$; hence, $\|T\|=\|T(\mathbf{1})\|=\left\|B_{0}\right\|$. To finish the proof of the basic implication $(b) \Rightarrow(a)$, we only have to show that $T(\varphi) \leq$ $\int_{\sigma(A)} \varphi(t) d E_{A}, \varphi \in(C(\sigma(A)))_{+},\|T\| \leq 1$.

Let $\varphi \in(C(\sigma(A)))_{+}$. According to Lemma 2, where $K$ stands for $\sigma(A)$, there exists a sequence $\left(\widetilde{p}_{l}\right)_{l \in \mathbb{N}}$ of polynomial functions such that $\widetilde{p}_{l} \geq \varphi_{0} \geq \mathbf{0}$ on $\mathbb{R}_{+},\left.\widetilde{p}_{l}\right|_{\sigma(A)} \rightarrow \varphi, l \rightarrow \infty$, 
uniformly on $\sigma(A)$. The continuity of $T$ and of $T_{2}: C(\sigma(A)) \rightarrow Y(A), T_{2}(g)=\int_{\sigma(A)} g d_{E_{A}}$, also using the last property on the sequence $\left(B_{j}\right)_{j \in \mathbb{N}}$ stated at (b), lead to:

$$
T(\varphi)=\lim _{l} T\left(\widetilde{p}_{l}\right) \leq \lim _{l} T_{2}\left(\widetilde{p}_{l}\right)=T_{2}(\varphi)=\int_{\sigma(A)} \varphi d_{E_{A}} .
$$

In particular, for $\varphi=\mathbf{1}$, we have: $T(\mathbf{1}) \leq \int_{\sigma(A)} 1 d_{E_{A}}=I \Rightarrow\|T\|=\|T(\mathbf{1})\| \leq\|I\|=1$. Here, $I: H \rightarrow H$ is the identity operator; this proves $(b) \Rightarrow(a)$. It is worth noting that we have used the expression of non-negative polynomials on $\mathbb{R}_{+}: p(t) \geq 0 \forall t \in \mathbb{R}_{+} \Leftrightarrow$ $p(t)=p_{1}^{2}(t)+t p_{2}^{2}(t), t \in \mathbb{R}_{+}$for some $p_{1}, p_{2} \in \mathbb{R}[t]$. The implication $(a) \Rightarrow(b)$ is obvious.

Next, we generalize the sandwich condition $0 \leq T \leq T_{2}$ on $X_{+}$, appearing in Corollary 1 (where $X=C(\sigma(A))$ ), to the condition $T_{1} \leq T \leq T_{2}$ on $X_{+}$, where $T_{1}, T_{2}$ are two given linear operators on $X, 0 \leq T_{1} \leq T_{2}$ on $X_{+}$. We start with a general result. Let $K \subset \mathbb{R}_{+}$be an arbitrary compact subset. We denote by $X=C(K)$ the Banach lattice of all real-valued continuous functions on $K$, and let $Y$ be an arbitrary order complete Banach lattice.

Theorem 7 (see [28], Theorem 3). Let $T_{1}, T_{2}$ be two linear operators from $X$ to $Y$ such that $\mathbf{0} \leq T_{1} \leq T_{2}$ on the positive cone of $X$, while $\left(y_{n}\right)_{n \geq 0}$ is a given sequence of elements in $Y$. The following statements are equivalent:

(a) There exists a unique (bounded) linear operator $T: X \rightarrow Y$, such that

$$
T\left(\varphi_{j}\right)=y_{j}, j \in \mathbb{N}, T_{1} \leq T \leq T_{2} \text { on the positive cone of } X,\left\|T_{1}\right\| \leq\|T\| \leq\left\|T_{2}\right\| ;
$$

(b) For any polynomial $\sum_{j=0}^{m} \alpha_{j} \varphi_{j} \geq \mathbf{0}$ on $K$, we have $\sum_{j=0}^{m} \alpha_{j} T_{1}\left(\varphi_{j}\right) \leq \sum_{j=0}^{m} \alpha_{j} y_{j}$; if $J_{0} \subset \mathbb{N}$ is a finite subset, and $\left\{\lambda_{j} ; j \in J_{0}\right\} \subset \mathbb{R}$, then the following conditions are satisfied:

$$
\sum_{i, j \in J_{0}} \lambda_{i} \lambda_{j} y_{i+j+l} \leq \sum_{i, j \in J_{0}} \lambda_{i} \lambda_{j} T_{2}\left(\varphi_{i+j+l}\right), l \in\{0,1\}
$$

(c) $T_{1} \leq T_{2}$ on $X_{+}$, and for any polynomial $\sum_{j \in J_{0}} \lambda_{j} \varphi_{j}$, the following inequality holds:

$$
\sum_{j \in J_{0}} \lambda_{j} y_{j} \leq T_{2}\left(\left(\sum_{j \in J_{0}} \lambda_{j} \varphi_{j}\right)^{+}\right)-T_{1}\left(\left(\sum_{j \in J_{0}} \lambda_{j} \varphi_{j}\right)^{-}\right) .
$$

Proof. According to the notations and assertions of (a), the implication $(a) \Rightarrow(b)$ is clear. To prove the converse implication, we observe that the first assertion of (b) says that in defining the following:

$$
T_{0}\left(\sum_{j=0}^{m} \alpha_{j} \varphi_{j}\right)=\sum_{j=0}^{m} \alpha_{j} y_{j}, m \in \mathbb{N}, \alpha_{j} \in \mathbb{R},
$$

we obtain a linear operator defined on the subspace of polynomial functions, which verifies the moment conditions:

$$
T_{0}\left(\varphi_{j}\right)=y_{j}, j \in \mathbb{N},
$$

( $T_{0} \geq T_{1}$ holds on the convex cone $\mathcal{P}_{+}$of all polynomial functions which are non-negative on $K)$. On the other hand, any element from $X=C(K)$ is dominated by a constant function, so that the subspace $\mathcal{P}$ of polynomial functions defined on $\mathbb{R}_{+}$verifies the hypothesis of Theorem 5, where $X_{1}$ stands for $X$, and $X_{0}$ stands for $\mathcal{P}$. According to Theorem 5, the linear operator $T_{0}-T_{1}: \mathcal{P} \rightarrow Y$, which is positive on $\mathcal{P}_{+}=\mathcal{P} \cap X_{+}$, admits a positive 
linear extension $U: X \rightarrow Y$. We define $T=T_{1}+U \geq T_{1}$ on $X_{+}$. In addition, $T \in L_{+}(X, Y)$ verifies the following:

$$
T\left(\varphi_{j}\right)=T_{1}\left(\varphi_{j}\right)+U\left(\varphi_{j}\right)=T_{1}\left(\varphi_{j}\right)+T_{0}\left(\varphi_{j}\right)-T_{1}\left(\varphi_{j}\right)=T_{0}\left(\varphi_{j}\right)=y_{j}, j \in \mathbb{N} .
$$

In other words, $T: X \rightarrow Y$ is a linear extension of $T_{0}: \mathcal{P} \rightarrow Y$, which dominates $T_{1}$ on $X_{+}$. Next, we prove that $T \leq T_{2}$ on $X_{+}$. To this end, observe that according to the second assertion of (b), we already know that $T \leq T_{2}$ on special polynomial functions, which are non-negative on the entirety of the semi-axes $\mathbb{R}_{+}$. Indeed, any non-negative polynomial $p=p(t)$ on $\mathbb{R}_{+}$has the explicit form $p(t)=q^{2}(t)+t r^{2}(t)$ for some $q, r \in \mathbb{R}[t]$. On the other hand, since

$$
T \geq T_{1} \geq \mathbf{0},
$$

the linear operator $T$ is positive and, hence, is also continuous; $T_{2}$ is continuous as well, thanks to its positivity. We now apply Lemma 2 for an arbitrary $\varphi \in X_{+}$. Using the notations of Lemma 2, and the above-discussed assertions, we infer the following:

$$
\mathbf{0} \leq T_{1}(\varphi) \leq T(\varphi)=\lim _{l \rightarrow \infty} T\left(\widetilde{p}_{l}\right) \leq \lim _{l \rightarrow \infty} T_{2}\left(\widetilde{p}_{l}\right)=T_{2}(\varphi), \varphi \in X_{+} .
$$

It remains to prove the last relation of (a). If $\psi$ is an arbitrary function in $X$, then the preceding inequality yields

$$
T(\psi) \leq T(|\psi|) \leq T_{2}(|\psi|)
$$

and, similarly, $-T(\psi)=T(-\psi) \leq T_{2}(|\psi|)$. These inequalities yield $|T(\psi)| \leq T_{2}(|\psi|)$ and, since $Y$ is a Banach lattice, the conclusion is $\|T(\psi)\| \leq\left\|T_{2}(|\psi|)\right\| \leq\left\|T_{2}|\||| \psi \mid\|=\right.$ $\left\|T_{2}\left|\||| \psi\|, \psi\right.\right.$ in $X$. Thus, $\|T\| \leq\left\|T_{2}\right\|$. Similarly, $\left\|T_{1}\right\| \leq\|T\|$. The equivalence $(a) \Leftrightarrow(c)$ follows directly from Theorem 2 . This completes the proof.

Corollary 2. With the notation of Corollary 1, assume that $A$ is a positive self-adjoint operator acting on $H$. Let $\alpha \in[0, \infty]$ be an arbitrary constant. The following statements are equivalent:

(a) There exists a unique positive linear operator $T: C(\sigma(A)) \rightarrow Y$, such that

$$
\begin{gathered}
T\left(\varphi_{j}\right)=B_{j}, j \in \mathbb{N}, \int_{\sigma(A)} \exp (-\alpha t) \varphi(t) d E_{A} \leq T(\varphi) \leq \int_{\sigma(A)} \varphi(t) d E_{A} \\
\forall \varphi \in(C(\sigma(A)))_{+},\|\exp (-\alpha A)\| \leq\|T\| \leq 1
\end{gathered}
$$

(b) For any polynomial $\sum_{j=0}^{m} \alpha_{j} t^{j} \geq 0 \forall t \in \sigma(A)$, we have

$$
\sum_{j=0}^{m} \alpha_{j} B_{j} \geq\left(\sum_{j=0}^{m} \alpha_{j} A^{j}\right) \exp (-\alpha A) ;
$$

if $J_{0} \subset \mathbb{N}$ is a finite subset, and $\left\{\lambda_{j} ; j \in J_{0}\right\} \subset \mathbb{R}$, then the following inequalities hold:

$$
\sum_{i, j \in J_{0}} \lambda_{i} \lambda_{j} B_{i+j+l} \leq \sum_{i, j \in J_{0}} \lambda_{i} \lambda_{j} A^{i+j+l}, l \in\{0,1\} .
$$

Proof. One applies Theorem 7, where $K$ stands for $\sigma(A)$,

$$
T_{1}(\varphi)=\int_{\sigma(A)} \exp (-\alpha t) \varphi(t) d E_{A}, T_{2}(\varphi)=\int_{\sigma(A)} \varphi(t) d E_{A}, \varphi \in C(\sigma(A)) .
$$

We observe that $T_{1}(\varphi)=\exp (-\alpha A) \varphi(A) \in\left[\mathbf{0}, T_{2}(\varphi)\right]$, for all $\varphi \in(C(\sigma(A)))_{+}$, since $t \geq 0, \varphi(t) \geq 0$, for all $t \in \sigma(A), \exp (-\alpha A) \in[\mathbf{0}, I]$ and the algebra $Y(A)$ is commutative. Moreover, as in the proof of Corollary 1 , 


$$
\left\|T_{1}\right\|=\left\|T_{1}(\mathbf{1})\right\|=\left\|\int_{\sigma(A)} \exp (-\alpha t) d E_{A}\right\|=\|\exp (-\alpha A)\| .
$$

On the other hand, the first condition of (b) says that

$$
\sum_{j=0}^{m} \alpha_{j} B_{j} \geq \sum_{j=0}^{m} \alpha_{j} T_{1}\left(\varphi_{j}\right)
$$

The second condition of (b) is exactly the second condition of (b) written in Theorem 7 , where $y_{j}$ stands for $B_{j}, j \in \mathbb{N}$. The conclusion follows via Theorem 7 .

Next, we review the key polynomial approximation result of this subsection, which works on arbitrary closed subsets $F \subseteq \mathbb{R}^{n}$.

Lemma 3 (see [28]). Let $F \subseteq \mathbb{R}^{n}$ be an unbounded closed subset, and $v$ an $M$-determinate measure on $F$ (with finite moments of all natural orders). Then, for any $x \in C_{0}(F), x(t) \geq 0, \forall t \in F$, there exists a sequence $\left(p_{m}\right)_{m}, p_{m} \geq x, m \in \mathbb{N}, p_{m} \rightarrow x$ in $L_{v}^{1}(F)$. In particular, we have

$$
\lim _{m} \int_{F} p_{m}(t) d v=\int_{F} x(t) d v
$$

$\mathcal{P}_{+}$is dense in $\left(L_{v}^{1}(F)\right)_{+}$, and $\mathcal{P}$ is dense in $L_{v}^{1}(F)$.

Proof. To prove the assertions of the statement, it is sufficient to show that for any $x \in$ $\left(C_{0}(F)\right)_{+}$, we have

$$
Q_{1}(x):=\inf \left\{\int_{F} p(t) d v ; p \geq x, p \in \mathcal{P}\right\}=\int_{F} x(t) d v
$$

Obviously, one has

$$
Q_{1}(x) \geq \int_{F} x(t) d v
$$

To prove the converse, we define the linear form

$$
T_{0}: X_{0}:=\mathcal{P} \oplus S p\{x\} \rightarrow \mathbb{R}, F_{0}(p+\alpha x):=\int_{F} p(t) d v+\alpha Q_{1}(x), p \in \mathcal{P}, \alpha \in \mathbb{R} .
$$

Next, we show that $F_{0}$ is positive on $X_{0}$. In fact, for $\alpha<0$, one has (from the definition of $Q_{1}$, which is a sublinear functional on $X_{1}$ ):

$$
p+\alpha x \geq 0 \Rightarrow p \geq-\alpha x \Rightarrow(-\alpha) Q_{1}(x)=Q_{1}(-\alpha x) \leq \int_{F} p(t) d v \Rightarrow T_{0}(p+\alpha x) \geq 0
$$

If $a \geq 0$, we infer that:

$$
\begin{aligned}
& 0=Q_{1}(0)=Q_{1}(\alpha x-\alpha x) \leq \alpha Q_{1}(x)+Q_{1}(-\alpha x) \Rightarrow \\
& \int_{F} p(t) d v \geq Q_{1}(-\alpha x) \geq-\alpha Q_{1}(x) \Rightarrow T_{0}(p+\alpha x) \geq 0
\end{aligned}
$$

where, in both possible cases, we have $x_{0} \in\left(X_{0}\right)_{+} \Rightarrow T_{0}\left(x_{0}\right) \geq 0$. Since $X_{0}$ contains the space of the polynomials' functions, which is a majorizing subspace of $X_{1}$, there exists a linear positive extension $T: X \rightarrow \mathbb{R}$ of $T_{0}$, which is continuous on $C_{0}(F)$ with respect to the sup-norm. Therefore, $T$ has a representation by means of a positive Borel regular measure $\mu$ on $F$, such that

$$
T(x)=\int_{F} x(t) d \mu, x \in C_{0}(F) .
$$


Let $p \in \mathcal{P}_{+}$be a non-negative polynomial function. There is a nondecreasing sequence $\left(x_{m}\right)_{m}$ of continuous non-negative function with compact support, such that $x_{m} \nearrow p$ pointwise on $F$. Positivity of $T$ and Lebesgue's dominated convergence theorem for $\mu$ yield

$$
\int_{F} p(t) d v=T(p) \geq \sup T\left(x_{m}\right)=\sup \int_{F} x_{m}(t) d \mu=\int_{F} p(t) d \mu, p \in \mathcal{P}_{+} .
$$

Thanks to Haviland's theorem, there exists a positive Borel regular measure $\lambda$ on $F$, such that

$$
\lambda(p)=v(p)-\mu(p) \Leftrightarrow v(p)=\lambda(p)+\mu(p), p \in \mathcal{P} .
$$

Since $v$ is assumed to be $M$-determinate, it follows that

$$
v(B)=\lambda(B)+\mu(B)
$$

for any Borel subset $B$ of $F$. From this last assertion, approximating each $x \in\left(L_{v}^{1}(F)_{+}\right.$, by a nondecreasing sequence of non-negative simple functions, and also using Lebesgue's convergence theorem, one obtains firstly for positive functions, then for arbitrary $v$-integrable functions, $\varphi$ :

$$
\int_{F} \varphi d v=\int_{F} \varphi d \lambda+\int_{F} \varphi d \mu, \varphi \in L_{v}^{1}(F) .
$$

In particular, we must have

$$
\int_{F} x d v \geq \int_{F} x d \mu=T(x)=T_{0}(x)=Q_{1}(x) .
$$

Then, Equations (6) and (7) conclude the proof.

Remark 2. We recall that the preceding Lemma 3 is no more valid when we replace $L_{v}^{1}(F)$ with the Hilbert space $L_{v}^{2}(F), F=\mathbb{R}^{n}, n \geq 2$ (see [13], Theorem 4.4, where the authors construct such a measure $v$ ).

The next theorem follows from Lemma 3. It facilitates proving inequalities on the entire $L_{v}^{1}(F)$ space verifying the involved inequalities only on polynomial functions (here $v$ is a moment determinate measure on $F$ ).

Theorem 8 (see [27], Theorem 2). Let $F$ be a closed unbounded subset of $\mathbb{R}^{n}, Y$ an order complete Banach lattice, $\left(y_{j}\right)_{j \in \mathbb{N}^{n}}$ a given sequence in $Y$, and $v$ an $M$-determinate measure on F. Let $T_{2} \in B\left(L_{v}^{1}(F), Y\right)$ be a linear positive (bounded) operator from $L_{v}^{1}(F)$ to $Y$. The following statements are equivalent:

(a) There exists a unique linear operator $T \in B\left(L_{v}^{1}(F), Y\right)$, such that $T\left(\varphi_{j}\right)=y_{j}, j \in \mathbb{N}^{n}$, $\mathbf{0} \leq T \leq T_{2}$ on the positive cone of $L_{v}^{1}(F)$, and $T \leq T_{2}$;

(b) For any finite subset $J_{0} \subset \mathbb{N}^{n}$, and any $\left\{a_{j}\right\}_{j \in J_{0}} \subset \mathbb{R}$, we have

$$
\sum_{j \in J_{0}} a_{j} \varphi_{j} \geq 0 F \Rightarrow \mathbf{0} \leq \sum_{j \in J_{0}} a_{j} y_{j} \leq \sum_{j \in J_{0}} a_{j} T_{2}\left(\varphi_{j}\right) .
$$

Proof. Observe that the assertion (b) says that

$$
0 \leq T_{0}(p) \leq T_{2}(p), p \in \mathcal{P}_{+},
$$

where $T_{0}: \mathcal{P} \rightarrow Y$ is the unique linear operator that verifies the interpolation conditions of Equation (1). Thus, $(a) \Rightarrow(b)$ is obvious. To prove the converse, consider the vector subspace $X_{1} \subset L_{v}^{1}(F)$ of all functions $\varphi \in L_{v}^{1}(F)$, verifying

$$
|\varphi(t)| \leq p(t) \forall t \in F
$$


for some polynomial $p$. Clearly, $X_{1}$ contains the subspace of polynomials as well as the subspace of continuous compactly supported real-valued functions. On the other hand, the subspace of polynomials is a majorizing subspace in $X_{1}$, and according to the first inequality of Equation (8), $T_{0}$ is positive as a linear operator on $\mathcal{P}$. Application of Theorem 5 yields the existence of a positive linear extension $T: X_{1} \rightarrow Y$ of $T_{0}$. Let $x$ be a non-negative continuous compactly supported function on $F$, and $\left(p_{m}\right)_{m}$ a sequence of polynomials with the properties specified in Lemma 3. According to the second inequality of Equation (8), we have

$$
T\left(p_{m}\right)=T_{0}\left(p_{m}\right) \leq T_{2}\left(p_{m}\right) \text { for all } m \in \mathbb{N} .
$$

Our next goal is to prove that

$$
T(x) \leq T_{2}(x) .
$$

Assuming the contrary, we should have $T_{2}(x)-T(x) \notin Y_{+}$. Since $Y_{+}$is closed, a Hahn-Banach separation theorem leads to the existence of a positive linear form $y^{\star}$ in the dual $Y^{\star}$ of $Y$, verifying

$$
y^{\star}\left(T_{2}(x)-T(x)\right)<0
$$

The positive linear form $y^{\star} \circ T$ has a representing positive regular Borel measure $\mu$, for which Fatou's lemma can be applied; we infer that

$$
y^{\star}(T(x)) \leq \operatorname{limin} f_{m} y^{\star}\left(T\left(p_{m}\right)\right) \leq \operatorname{limin} f_{m} y^{\star}\left(T_{2}\left(p_{m}\right)\right)=y^{\star}\left(T_{2}\left(\lim _{m} p_{m}\right)\right)=y^{\star}\left(T_{2}(x)\right) .
$$

Equations (10) and (11) yield

$$
y^{\star}\left(T_{2}(x)\right)<y^{\star}(T(x)) \leq y^{\star}\left(T_{2}(x)\right),
$$

implying the contradiction $y^{\star}\left(T_{2}(x)\right)<y^{\star}\left(T_{2}(x)\right)$. Hence, the assumption $T_{2}(x)-T(x) \notin$ $Y_{+}$was false, such that we must have $T_{2}(x)-T(x) \in Y_{+}$, i.e., Equation (9) is proven. Now, let $\varphi \in C_{0}(F)$ be arbitrary. According to the preceding considerations, we obtain

$$
|T(\varphi)|=\left|T\left(\varphi^{+}\right)-T\left(\varphi^{-}\right)\right| \leq T\left(\varphi^{+}\right)+T\left(\varphi^{-}\right) \leq T_{2}\left(\varphi^{+}\right)+T_{2}\left(\varphi^{-}\right)=T_{2}(|\varphi|) .
$$

Since the norm on $Y$ is solid $\left(\left|y_{1}\right| \leq\left|y_{2}\right| \Rightarrow|| y_{1}|| \leq|| y_{2}||\right)$, we infer that

$$
\|T(\varphi)\| \leq\left\|T_{2}(|\varphi|)\right\| \leq\left\|T_{2}\right\|\|\| \varphi\|\|_{1}=\left\|T_{2}\right\|\left\|\mid \varphi_{1}\right\|, \forall \varphi \in C_{0}(F) .
$$

Using the fact that $C_{0}(F)$ is dense in $L_{v}^{1}(F)$ (see [9]), the last evaluation leads to the existence of a linear extension $\widetilde{T}: L_{v}^{1}(F) \rightarrow Y$ of $T$, such that

$$
\|\widetilde{T}(\varphi)\| \leq\left\|T_{2}\left|\left\||| \varphi_{1}\right\|, \quad\|\varphi\|_{1}=\int_{A}\right| \varphi(t) \mid d v, \varphi \in L_{v}^{1}(F) .\right.
$$

It follows that $\|\widetilde{T}|| \leq\| T_{2} \|$, and the positivity of $\widetilde{T}$ is a consequence of the positivity of $T$, via continuity of the extension $\widetilde{T}$ and the density of $\left(C_{0}(F)\right)_{+}$in $\left(L_{v}^{1}(F)\right)_{+}$. We also note that

$$
\widetilde{T}\left(\varphi_{j}\right)=T_{0}\left(\varphi_{j}\right)=y_{j}, j \in \mathbb{N}^{n} .
$$

This concludes the proof.

Unlike the case of nonnegative polynomials on $\mathbb{R}^{2}$, the nonnegative polynomials on a strip are expressible in terms of sums of squares, due to the following result of M. Marshall. 
Theorem 9 (see [18]). Supposing that $p\left(t_{1}, t_{2}\right) \in \mathbb{R}\left[t_{1}, t_{2}\right]$ is non-negative on the strip $F=$ $[0,1] \times \mathbb{R}$, then $p\left(t_{1}, t_{2}\right)$ is expressible as

$$
p\left(t_{1}, t_{2}\right)=\sigma\left(t_{1}, t_{2}\right)+\tau\left(t_{1}, t_{2}\right) t_{1}\left(1-t_{1}\right)
$$

where $\sigma\left(t_{1}, t_{2}\right), \tau\left(t_{1}, t_{2}\right)$ are sums of squares in $\mathbb{R}\left[t_{1}, t_{2}\right]$.

Let $F=[0,1] \times \mathbb{R}, v$ a M-determinate measure on $F$, and $X=L_{v}^{1}(F), \varphi_{j}\left(t_{1}, t_{2}\right):=$ $t_{1}^{j_{1}} t_{2}^{j_{2}}, j=\left(j_{1}, j_{2}\right) \in \mathbb{N}^{2},\left(t_{1}, t_{2}\right) \in F$. Let $Y$ be an order complete Banach lattice, and $\left(y_{j}\right)_{j \in \mathbb{N}^{2}}$ a sequence of given elements in $Y$. The next result follows directly from Theorems 8 and 9 .

Theorem 10. Let $T_{2} \in B_{+}(X, Y)$ be a linear (bounded) positive operator from $X$ to $Y$. The following statements are equivalent:

(a) There exists a unique (bounded) linear operator $T: X \rightarrow Y$, such that $T\left(\varphi_{j}\right)=y_{j}, j \in \mathbb{N}^{2}$, where $T$ is between zero and $T_{2}$ on the positive cone of $X,\|T\| \leq\left\|T_{2}\right\| ; ;$

(b) For any finite subset $J_{0} \subset \mathbb{N}^{2}$, and any $\left\{\lambda_{j} ; j \in J_{0}\right\} \subset \mathbb{R}$,we have:

$$
\begin{gathered}
0 \leq \sum_{i, j \in J_{0}} \lambda_{i} \lambda_{j} y_{i+j} \leq \sum_{i, j \in J_{0}} \lambda_{i} \lambda_{j} T_{2}\left(\varphi_{i+j}\right) ; \\
0 \leq \sum_{i, j \in J_{0}} \lambda_{i} \lambda_{j}\left(y_{i_{1}+j_{1}+1, i_{2}+j_{2}}-y_{i_{1}+j_{1}+2, i_{2}+j_{2}}\right) \leq \\
\sum_{i, j \in J_{0}} \lambda_{i} \lambda_{j}\left(T_{2}\left(\varphi_{i_{1}+j_{1}+1, i_{2}+j_{2}}-\varphi_{i_{1}+j_{1}+2, i_{2}+j_{2}}\right)\right), i=\left(i_{1}, i_{2}\right), j=\left(j_{1}, j_{2}\right) \in J_{0} .
\end{gathered}
$$

Lemma 4 (see [28], Lemma 4). Let $v=v_{1} \times \cdots \times v_{n}$ be a product of $n M$-determinate measures on $\mathbb{R}_{+}=[0, \infty[$. Then we can approximate any non-negative continuous compactly supported function in $X=L_{v}^{1}\left(\mathbb{R}_{+}^{n}\right)$ with sums of products

$$
p_{1} \otimes \cdots \otimes p_{n}
$$

$p_{j}$ positive polynomial on the real non-negative semi-axis, in variable $t_{j} \in \mathbb{R}_{+}, j=1, \ldots, n$, where

$$
\left(p_{1} \otimes \cdots \otimes p_{n}\right)\left(t_{1}, \ldots, t_{n}\right)=p_{1}\left(t_{1}\right) \cdots p_{n}\left(t_{n}\right) .
$$

The idea of the proof is to use Bernstein approximating polynomials of $n$ variables, and then to apply Lemma 3 to the case $n=1, F=\mathbb{R}_{+}$, for each moment determinate measure $v_{j}, j=1, \ldots, n$. By means of the same arguments, the next result holds true as well:

Lemma 5. Let $v=v_{1} \times \cdots \times v_{n}$ be a product of $n M$-determinate measures on $\mathbb{R}$. Then, we can approximate any non-negative continuous compactly supported function in $X=L_{v}^{1}\left(\mathbb{R}^{n}\right)$ with sums of products

$$
p_{1} \otimes \cdots \otimes p_{n}
$$

$p_{j}$ non-negative polynomial on the entire real line $, j=1, \ldots, n$.

Corollary 3 (see [28], Theorem 5). Let $X$ be as in Lemma $4,\left(y_{j}\right)_{j \in \mathbb{N}^{n}}$ a sequence in $Y$, where $Y$ is an order complete Banach lattice; and let $T_{2} \in B_{+}(X, Y)$ be a positive bounded linear operator. The following statements are equivalent:

(a) There exists a unique (bounded) linear operator $T \in B(X, Y)$, such that $T\left(\varphi_{j}\right)=y_{j}, j \in \mathbb{N}^{n}$; $T$ is between zero and $T_{2}$ on the positive cone of $X,\|T\| \leq\left\|T_{2}\right\|$;

(b) For any finite subset $J_{0} \subset \mathbb{N}^{n}$, and any $\left\{\lambda_{j} ; j \in J_{0}\right\} \subset \mathbb{R}$, we have

$$
\sum_{j \in J_{0}} \lambda_{j} \varphi_{j}(t) \geq 0 \forall t \in \mathbb{R}_{+}^{n} \Rightarrow \sum_{j \in j_{0}} \lambda_{j} y_{j} \in Y_{+} .
$$


For any finite subsets $J_{k} \subset \mathbb{N}, k=1, \ldots, n$, and any $\left\{\lambda_{j_{k}}\right\}_{j_{k} \in J_{k}} \subset \mathbb{R}, k=1, \ldots, n$, the following relations hold:

$$
\begin{gathered}
\sum_{i_{1}, j_{1} \in J_{1}}\left(\cdots\left(\sum_{i_{n}, j_{n} \in J_{n}} \lambda_{i_{1}} \lambda_{j_{1}} \ldots \lambda_{i_{n}} \lambda_{j_{n}} y_{i_{1}+j_{1}+l_{1}, \ldots, i_{n}+j_{n}+l_{n}}\right) \cdots\right) \leq \\
\sum_{i_{1}, j_{1} \in J_{1}}\left(\cdots\left(\sum_{i_{n}, j_{n} \in J_{n}} \lambda_{i_{1}} \lambda_{j_{1}} \ldots \lambda_{i_{n}} \lambda_{j_{n}} T_{2}\left(\varphi_{i_{1}+j_{1}+l_{1}, \ldots, i_{n}+j_{n}+l_{n}}\right) \cdots\right),\left(l_{1}, \ldots, l_{n}\right) \in\{0,1\}^{n} .\right.
\end{gathered}
$$

Proof. One repeats the proof of Theorem 8 , where the convergent sequence of non-negative polynomials for the continuous non-negative compactly supported function $x$ can be chosen in terms of sums of tensor products of non-negative polynomials on $\mathbb{R}_{+}$. Such convergent sequences do exist, as shown by the preceding Lemma 4 . The last inequality of (b) in the present corollary says that on each term $p$ of such a sequence, the second inequality of Equation (8) holds true, where $\mathcal{P}_{+}$must be replaced by the convex cone $\mathcal{P}_{++}$of all special non-negative polynomials generated by the tensor products emphasized in Lemma 4 . The motivation of the condition $\left(l_{1}, \ldots, l_{n}\right) \in\{0,1\}^{n}$ of $(\mathrm{b})$ in the present statement comes from the fact that $p_{j}(t) \geq 0$ for all $t \in \mathbb{R}_{+} \Leftrightarrow p_{j}(t)=q_{j}^{2}(t)+t r_{j}^{2}(t), t \in \mathbb{R}_{+}$, for some $q_{j}, r_{j} \in \mathbb{R}[t]$. This ends the proof.

Corollary 4. Let $v$ be an $M$ - determinate measure on $\mathbb{R}_{+}$, with finite moments of all natural orders, $X=L_{v}^{1}\left(\mathbb{R}_{+}\right)$. Let $Y$ be an order complete Banach lattice, and $T_{2} \in B_{+}(X, Y),\left(y_{j}\right)_{j \in \mathbb{N}} a$ sequence in $Y$. The following statements are equivalent:

(a) There exists a unique (bounded) linear operator $T \in B(X, Y)$ that satisfies the conditions

$$
T\left(\varphi_{j}\right)=y_{j}, j \in \mathbb{N}, \mathbf{0} \leq T \leq T_{2} \text { on } X_{+},\|T\| \leq\left\|T_{2}\right\| ;
$$

(b) For any finite subset $J_{0} \subset \mathbb{N}$, and any $\left\{\lambda_{j} ; j \in J_{0}\right\} \subset \mathbb{R}$, the following inequalities hold true:

$$
0 \leq \sum_{i, j \in J_{0}} \lambda_{i} \lambda_{j} y_{i+j+k} \leq \sum_{i, j \in J_{0}} \lambda_{i} \lambda_{j} T_{2}\left(\varphi_{i+j+k}\right), k \in\{0,1\} .
$$

Similarly to Corollary 3, also using Lemma 5 and the fact that any non-negative polynomial on the real axes is the sum of squares of (two) polynomials, if we denote $\varphi_{j}(t):=t_{1}^{j_{1}} \cdots t_{n}^{j_{n}}, j=\left(j_{1}, \ldots, j_{n}\right) \in \mathbb{N}^{n}, t=\left(t_{1}, \ldots, t_{n}\right) \in \mathbb{R}^{n}$, then the following result holds true:

Corollary 5 (see [27], Theorem 6). Let $X=L_{v}^{1}\left(\mathbb{R}^{n}\right)$, where $v=v_{1} \times \cdots \times v_{n}$ is a product of $n M$-determinate measures on $\mathbb{R}$; let $Y$ be an order complete Banach lattice, and $\left(y_{j}\right)_{j \in \mathbb{N}^{n}}$ a sequence in $Y, T_{2} \in B_{+}(X, Y)$. The following statements are equivalent:

(a) There exists a unique (bounded) linear operator $T \in B(X, Y)$, such that $T\left(\varphi_{j}\right)=y_{j}, j \in$ $\mathbb{N}^{n}, \mathbf{0} \leq T \leq T_{2}$ on the positive cone of $X,\|T\| \leq\left\|T_{2}\right\| ;$

(b) For any finite subset $J_{0} \subset \mathbb{N}^{n}$, and any $\left\{\lambda_{j} ; j \in J_{0}\right\} \subset \mathbb{R}$, we have

$$
\sum_{j \in J_{0}} \lambda_{j} \varphi_{j}(t) \geq 0 \forall t \in \mathbb{R}^{n} \Rightarrow \sum_{j \in j_{0}} \lambda_{j} y_{j} \in Y_{+} .
$$


For any finite subsets $J_{k} \subset \mathbb{N}, k=1, \ldots, n$, and any $\left\{\lambda_{j_{k}}\right\}_{j_{k} \in J_{k}} \subset \mathbb{R}, k=1, \ldots, n$, the following relations hold:

$$
\begin{array}{r}
\sum_{i_{1}, j_{1} \in J_{1}}\left(\cdots\left(\sum_{i_{n}, j_{n} \in J_{n}} \lambda_{i_{1}} \lambda_{j_{1}} \ldots \lambda_{i_{n}} \lambda_{j_{n}} y_{i_{1}+j_{1}, \ldots, i_{n}+j_{n}}\right) \cdots\right) \leq \\
\sum_{i_{1}, j_{1} \in J_{1}}\left(\cdots\left(\sum_{i_{n}, j_{n} \in J_{n}} \lambda_{i_{1}} \lambda_{j_{1}} \ldots \lambda_{i_{n}} \lambda_{j_{n}} T_{2}\left(\varphi_{i_{1}+j_{1}, \ldots, i_{n}+j_{n}}\right) \cdots\right) .\right.
\end{array}
$$

Corollary 6. Let us consider the hypothesis and notations from Corollary 4 , where we replace $\mathbb{R}_{+}$ with $\mathbb{R}$. The following statements are equivalent:

(a) There exists a unique (bounded) linear operator $T \in B(X, Y)$, which verifies

$$
T\left(\varphi_{j}\right)=y_{j}, j \in \mathbb{N}, 0 \leq T \leq T_{2} \text { on } X_{+},\|T\| \leq\left\|T_{2}\right\| ;
$$

(b) For any finite subset $J_{0} \subset \mathbb{N}$, and any $\left\{\lambda_{j} ; j \in J_{0}\right\} \subset \mathbb{R}$, the following inequalities hold true:

$$
0 \leq \sum_{i, j \in J_{0}} \lambda_{i} \lambda_{j} y_{i+j} \leq \sum_{i, j \in J_{0}} \lambda_{i} \lambda_{j} T_{2}\left(\varphi_{i+j}\right)
$$

Theorem 11 (see [27]). Let $X$ be as in Corollary 5, and $Y$ be a Banach lattice. Assume that $T$ is a linear bounded operator from $X$ to $Y$. The following statements are equivalent:

(a) $T \geq \mathbf{0}$ on the positive cone of $X$;

(b) For any finite subsets $J_{k} \subset \mathbb{N}, k=1, \ldots, n$, and any $\left\{\lambda_{j_{k}}\right\}_{j_{k} \in J_{k}} \subset \mathbb{R}, k=1, \ldots, n$, the following relations hold:

$$
\mathbf{0} \leq \sum_{i_{1}, j_{1} \in J_{1}}\left(\cdots\left(\sum_{i_{n} . j_{n} \in J_{n}} \lambda_{i_{1}} \lambda_{j_{1}} \cdots \lambda_{i_{n}} \lambda_{j_{n}} T\left(x_{i_{1}+j_{1}}, \cdots, i_{n}+j_{n}\right)\right) \cdots\right)
$$

Proof. Note that (b) says that $T$ is positive on the convex cone generated by special positive polynomials $p_{1} \otimes \cdots \otimes p_{n}$, each factor of any term in the sum being non-negative on the whole real axis. Consequently, $(a) \Rightarrow(b)$ is clear. In order to prove the converse, observe that any non-negative element of $X$ can be approximated by non-negative continuous compactly supported functions. Such functions can be approximated by the sums of tensor products of positive polynomials in each separate variable, the latter being sums of squares. The conclusion is that any non-negative function from $X$ can be approximated in $X=L_{v}^{1}\left(\mathbb{R}^{n}\right)$ by the sums of tensor products of squares of polynomials in each separate variable. We know that on such special polynomials, $T$ admits values in $Y_{+}$, according to condition (b). Now, the desired conclusion is a consequence of the continuity of $T$, also using the fact that the positive cone of $Y$ is closed. This concludes the proof.

In what follows, we review some of the results of [28]. If $F \subseteq \mathbb{R}^{n}$ is an arbitrary closed unbounded subset, then we denote by $\mathcal{P}_{+}$the convex cone of all polynomial functions (with real coefficients), taking non-negative values at any point of $F$. $\mathcal{P}_{++}$will be a subcone of $\mathcal{P}_{+}$, generated by special non-negative polynomials expressible in terms of sums of squares.

Theorem 12 (see [28]). Let $F \subseteq \mathbb{R}^{n}$ be a closed unbounded subset; $v$ a moment-determinate measure on $F$, having finite moments of all orders; and $X=L_{v}^{1}(F), \varphi_{j}(t)=t^{j}, t \in F, j \in \mathbb{N}^{n}$. Let $Y$ be an order complete Banach lattice, $\left(y_{j}\right)_{j \in \mathbb{N}^{n}}$ a given sequence of elements in $Y, T_{1}$ and $T_{2}$ two bounded linear operators from $X$ to $Y$. Assume that there exists a sub-cone $\mathcal{P}_{++} \subseteq \mathcal{P}_{+}$, such that each $f \in\left(C_{0}(F)\right)_{+}$can be approximated in $X$ by a sequence $\left(p_{l}\right)_{l}, p_{l} \in \mathcal{P}_{++}, p_{l} \geq f$ for all $l$. The following statements are equivalent:

(a) There exists a unique (bounded) linear operator $T: X \rightarrow Y, T\left(\varphi_{j}\right)=y_{j}, j \in \mathbb{N}^{n}$, $0 \leq T_{1} \leq T \leq T_{2}$ on $X_{+},\left\|T_{1}\right\| \leq\|T\| \leq\left\|T_{2}\right\| ;$ 
(b) For any finite subset $J_{0} \subset \mathbb{N}^{n}$, and any $\left\{\lambda_{j} ; j \in J_{0}\right\} \subset \mathbb{R}$, the following implications hold true:

$$
\begin{gathered}
\sum_{j \in J_{0}} \lambda_{j} \varphi_{j} \in \mathcal{P}_{+} \Rightarrow \sum_{j \in J_{0}} \lambda_{j} T_{1}\left(\varphi_{j}\right) \leq \sum_{j \in J_{0}} \lambda_{j} y_{j} \\
\sum_{j \in J_{0}} \lambda_{j} \varphi_{j} \in \mathcal{P}_{++} \Rightarrow \sum_{j \in J_{0}} \lambda_{j} T_{1}\left(\varphi_{j}\right) \geq \mathbf{0}, \sum_{j \in J_{0}} \lambda_{j} y_{j} \leq \sum_{j \in J_{0}} \lambda_{j} T_{2}\left(\varphi_{j}\right) .
\end{gathered}
$$

Proof. We start by observing that the first condition of Equation (13) implies the positivity of the bounded linear operator $T_{1}$, via its continuity. Indeed, if $f \in\left(C_{0}(F)\right)_{+}, p_{l} \in \mathcal{P}_{++}, p_{l} \geq$ $f$ for all $l, p_{l} \rightarrow f$ in $L_{v}^{1}(F)$ then, according to the first condition of Equation (13), $T_{1}\left(p_{l}\right) \geq$ 0 for all $l \in \mathbb{N}$, and the continuity of $T_{1}$ yields the following:

$$
T_{1}(f)=\lim _{l} T_{1}\left(p_{l}\right) \geq \mathbf{0}
$$

Since $\left(C_{0}(F)\right)_{+}$is dense in $X_{+}$as explained by measure theory, the continuity of $T_{1}$ implies $T_{1} \geq \mathbf{0}$ on $X_{+}$. Thus, $T_{1}$ is a positive linear operator. Next, we define $T_{0}: \mathcal{P} \rightarrow Y, T_{0}\left(\sum_{j \in J_{0}} \lambda_{j} \varphi_{j}\right)=\sum_{j \in J_{0}} \lambda_{j} y_{j}$, where the sums are finite and the coefficients $\lambda_{j}$ are arbitrary real numbers. Equation (12) says that $T_{0}-T_{1} \geq 0$ on $\mathcal{P}_{+}$. If we consider the vector subspace $X_{1}$ of $X$ formed by all functions $\psi \in X$ having the modulus $|\psi|$ dominated by a polynomial $p \in \mathcal{P}_{+}$on the entire set $F$, then $\mathcal{P}$ is a majorizing subspace of $X_{1}$, and $T_{0}-T_{1}$ is a positive linear operator on $\mathcal{P}$. The application of Theorem 5 leads to the existence of a positive linear extension $U: X_{1} \rightarrow Y$ of $T_{0}-T_{1}$. Clearly, $X_{1}$ contains $C_{0}(F)+\mathcal{P}$. Indeed, since $\varphi \in C_{0}(S) \Rightarrow|\varphi| \in\left(C_{0}(F)\right)_{+} \Rightarrow|\varphi| \leq b \mathbf{1} \in \mathcal{P}$ (according to Weierstrass' theorem), we infer that $\varphi \in X_{1}$; here, $b<\infty$ is a real number. Hence, $C_{0}(F) \subset X_{1}$. Now, if $p \in \mathcal{P}$, we observe the following:

$$
\mathbf{1}+p^{2}-2|p|=(\mathbf{1}-|p|)^{2} \geq 0,
$$

which can be written as follows:

$$
|p| \leq \frac{\mathbf{1}+p^{2}}{2} \in \mathcal{P}
$$

According to the definition of $X_{1}$, it results in $\mathcal{P} \subset X_{1}$. Consequently, $C_{0}(F)+\mathcal{P} \subset X_{1}$. Going back to the positive linear extension $U: X_{1} \rightarrow Y$ of $T_{0}-T_{1}$, we conclude that $\hat{T}_{0}=U+T_{1}: X_{1} \rightarrow Y$ is an extension of $T_{0}, \hat{T}_{0} \geq T_{1}$ on $\left(X_{1}\right)_{+}$, and $\hat{T}_{0}(p)=T_{0}(p) \leq T_{2}(p)$ for all $p \in \mathcal{P}_{++}$, according to the last requirement of Equation (13). A first conclusion is as follows:

$$
T_{1}(p) \leq \hat{T}_{0}(p) \leq T_{2}(p) \text { for all } p \in \mathcal{P}_{++}, \hat{T}_{0}(\psi) \geq T_{1}(\psi) \geq 0, \psi \in\left(X_{1}\right)_{+} .
$$

Our next goal is to prove the continuity of $\hat{T}_{0}$ on $C_{0}(S)$. Let $\left(f_{l}\right)_{l>0}$ be a sequence of non-negative continuous compactly supported functions, such that $f_{l} \rightarrow 0$ in $X_{1}$, and take a sequence of polynomials $p_{l} \geq f_{l} \geq 0, p_{l} \in \mathcal{P}_{++}$for all $l$, such that the following convergence result holds: $\left\|p_{l}-f_{l}\right\|_{1} \rightarrow 0, l \rightarrow \infty$. Then, apply the following:

$$
\left\|p_{l}\right\|_{1} \leq\left\|p_{l}-f_{l}\right\|_{1}+\left\|f_{l}\right\|_{1} \rightarrow 0, l \rightarrow \infty .
$$

Now, Equation (14) and the continuity of $T_{1}, T_{2}$ yield the following:

$$
0 \leftarrow T_{1}\left(p_{l}\right) \leq \hat{T}_{0}\left(p_{l}\right) \leq T_{2}\left(p_{l}\right) \rightarrow 0 .
$$

Hence, $\hat{T}_{0}\left(p_{l}\right) \rightarrow 0$; this results in the following:

$$
0 \leq T_{1}\left(f_{l}\right) \leq \hat{T}_{0}\left(f_{l}\right) \leq \hat{T}_{0}\left(p_{l}\right) \rightarrow 0 .
$$


Hence, $\hat{T}_{0}\left(f_{l}\right) \rightarrow 0$. If $\left(g_{n}\right)_{n \geq 0}$ is an arbitrary sequence of compactly supported and continuous functions, such that $g_{n} \rightarrow 0$ in $X_{1}$, then $g_{n}^{+} \rightarrow 0, g_{n}^{-} \rightarrow 0$. According to what we already have proven, we can write $\hat{T}_{0}\left(g_{n}^{+}\right) \rightarrow 0$ and $\hat{T}_{0}\left(g_{n}^{-}\right) \rightarrow 0$, which further yields $\hat{T}_{0}\left(g_{n}\right) \rightarrow 0$. This proves the continuity of $\hat{T}_{0}$ on $C_{0}(F)$, and the subspace $C_{0}(F)$ is dense in $X$. Hence, there exists a unique continuous linear extension $T \in B(X, Y)$ of $\hat{T}_{0}$. This results in $0 \leq T_{1} \leq T \leq T_{2}$ on $X_{+},\left\|T_{1}\right\| \leq\|T\| \leq\left\|T_{2}\right\|, T\left(\varphi_{j}\right)=T_{0}\left(\varphi_{j}\right)=y_{j}, j \in \mathbb{N}^{n}$. Indeed, $T_{1}, T, T_{2}$ are linear and continuous, and $\mathcal{P}_{++}$is dense in $\left(C_{0}(F)\right)_{+}$; hence, it is dense in $X_{+}$as well. For an arbitrary $\varphi \in X$, the following inequalities hold true, via the preceding remarks:

$$
\begin{gathered}
\pm T(\varphi)=T( \pm \varphi) \leq T(|\varphi|) \leq T_{2}(|\varphi|) \Rightarrow \\
|T(\varphi)| \leq T_{2}(|\varphi|) \Rightarrow\|T(\varphi)\| \leq\left\|T_{2}(|\varphi|)\right\| \leq\left\|T_{2}|\|||\varphi| \mid .\right.
\end{gathered}
$$

It follows that $\|T\| \leq\left\|T_{2}\right\|$ and, similarly, $\left\|T_{1}\right\| \leq\|T\|$. The uniqueness of the solution $T$ follows according to the density of polynomials in $X$, via the continuity of the linear operator $T$ and application of Lemma 3. This ends the proof.

Corollary 7 (see [28]). Let $v=v_{1} \times \cdots \times v_{n}, n \geq 2, v_{j}$ being an $M$-determinate (momentdeterminate) measure on $\mathbb{R}, j=1, \ldots, n, X=L_{v}^{1}\left(\mathbb{R}^{n}\right), \varphi_{j}(t)=t^{j}, t \in \mathbb{R}^{n}, j \in \mathbb{N}^{n}$. Additionally, assume that $v_{j}$ has finite moments of all orders, $j=1, \ldots, n$. Let $Y$ be an order complete Banach lattice, $\left(y_{j}\right)_{j \in \mathbb{N}^{n}}$ a given sequence of elements in $Y$, and $T_{1}$ and $T_{2}$ two bounded linear operators from $X$ to $Y$. The following statements are equivalent:

(a) There exists a unique (bounded) linear operator $T: X \rightarrow Y, T\left(\varphi_{j}\right)=y_{j}, j \in \mathbb{N}^{n}$, $\mathbf{0} \leq T_{1} \leq T \leq T_{2}$ on $X_{+}, T_{1} \leq T \leq T_{2}$;

(b) For any finite subset $J_{0} \subset \mathbb{N}^{n}$, and any $\left\{\lambda_{j} ; j \in J_{0}\right\} \subset \mathbb{R}$, the following implication holds true:

$$
\sum_{j \in J_{0}} \lambda_{j} \varphi_{j} \in \mathcal{P}_{+} \Rightarrow \sum_{j \in J_{0}} \lambda_{j} T_{1}\left(\varphi_{j}\right) \leq \sum_{j \in J_{0}} \lambda_{j} y_{j}
$$

For any finite subsets $J_{k} \subset \mathbb{N}, k=1, \ldots, n$, and any $\left\{\lambda_{j_{k}}\right\}_{j_{k} \in J_{k}} \subset \mathbb{R}$, the following inequalities hold true:

$$
\begin{aligned}
0 \leq & \sum_{i_{1}, j_{1} \in J_{1}}\left(\cdots\left(\sum_{i_{n} \cdot j_{n} \in J_{n}} \lambda_{i_{1}} \lambda_{j_{1}} \cdots \lambda_{i_{n}} \lambda_{j_{n}} T_{1}\left(\varphi_{i_{1}+j_{1}, \ldots, i_{n}+j_{n}}\right)\right) \cdots\right) ; \\
& \sum_{i_{1}, j_{1} \in J_{1}}\left(\cdots\left(\sum_{i_{n} \cdot j_{n} \in J_{n}} \lambda_{i_{1}} \lambda_{j_{1}} \cdots \lambda_{i_{n}} \lambda_{j_{n}} y_{i_{1}+j_{1}, \ldots, i_{n}+j_{n}}\right) \cdots\right) \leq \\
& \sum_{i_{1}, j_{1} \in J_{1}}\left(\cdots\left(\sum_{i_{n} \cdot j_{n} \in J_{n}} \lambda_{i_{1}} \lambda_{j_{1}} \cdots \lambda_{i_{n}} \lambda_{j_{n}} T_{2}\left(\varphi_{i_{1}+j_{1}, \ldots, i_{n}+j_{n}}\right)\right) \cdots\right) .
\end{aligned}
$$

Proof. One applies Theorem 12 and Lemma 5.

Corollary 8 (see [28]). Let $X=L_{v}^{1}(\mathbb{R})$, where $v$ is a moment-determinate measure on $\mathbb{R}$. Assume that $Y$ is an arbitrary order complete Banach lattice, and $\left(y_{n}\right)_{n \geq 0}$ is a given sequence with its terms in $Y$. Let $T_{1}, T_{2}$ be two linear operators from $X$ to $Y$, such that $\mathbf{0} \leq T_{1} \leq T_{2}$ on $X_{+}$. The following statements are equivalent:

(a) There exists a unique bounded linear operator $T$ from $X t o Y, T_{1} \leq T \leq T_{2}$ on $X_{+},\left\|T_{1}\right\| \leq$ $\|T\| \leq\left\|T_{2}\right\|$, such that $T\left(\varphi_{n}\right)=y_{n}$ for all $n \in \mathbb{N} ; ;$

(b) If $J_{0} \subset \mathbb{N}$ is a finite subset, and $\left\{\lambda_{j} ; j \in J_{0}\right\} \subset \mathbb{R}$, then

$$
\sum_{i, j \in J_{0}} \lambda_{i} \lambda_{j} T_{1}\left(\varphi_{i+j}\right) \leq \sum_{i, j \in J_{0}} \lambda_{i} \lambda_{j} y_{i+j} \leq \sum_{i, j \in J_{0}} \lambda_{i} \lambda_{j} T_{2}\left(\varphi_{i+j}\right)
$$


For $Y=\mathbb{R}$, based on the measure theory arguments discussed in [9], Corollary 8 can be written as follows:

Corollary 9. Letv be a moment determinate measure on $\mathbb{R}$. Assume that $h_{1}, h_{2}$ are two functions in $L_{v}^{\infty}(\mathbb{R})$, such that $0 \leq h_{1} \leq h_{2}$ almost everywhere. Let $\left(y_{n}\right)_{n \geq 0}$ be a given sequence of real numbers. The following statements are equivalent:

(a) There exists $h \in L_{v}^{\infty}(\mathbb{R})$, such that $h_{1} \leq h \leq h_{2} v$-almost everywhere, $\int_{\mathbb{R}} t^{j} h(t) d v=y_{j}$ for all $j \in \mathbb{N}$;

(b) If $J_{0} \subset \mathbb{N}$ is a finite subset, and $\left\{\lambda_{j} ; j \in J_{0}\right\} \subset \mathbb{R}$, then

$$
\sum_{i, j \in J_{0}} \lambda_{i} \lambda_{j} \int_{\mathbb{R}} t^{i+j} h_{1}(t) d v \leq \sum_{i, j \in J_{0}} \lambda_{i} \lambda_{j} y_{i+j} \leq \sum_{i, j \in J_{0}} \lambda_{i} \lambda_{j} \int_{\mathbb{R}} t^{i+j} h_{2}(t) d v .
$$

Similarly to Corollary 9 , replacing $\mathbb{R}$ with $\mathbb{R}_{+}$we can derive the following:

Corollary 10. Let $X=L_{v}^{1}\left(\mathbb{R}_{+}\right)$, where $v$ is a moment-determinate measure on $\mathbb{R}_{+}$. Assume that $Y$ is an arbitrary order complete Banach lattice, and $\left(y_{n}\right)_{n>0}$ is a given sequence with its terms in $Y$. Let $T_{1}, T_{2}$ be two linear operators from $X$ to $Y$, such that $0 \leq T_{1} \leq T_{2}$ on $X_{+}$. As usual, we denote $\varphi_{j}(t)=t^{j}, j \in \mathbb{N}, t \in \mathbb{R}_{+}$. The following statements are equivalent:

(a) There exists a unique bounded linear operator $T$ from $X$ to $Y, T_{1} \leq T \leq T_{2}$ on $X_{+}$, $T_{1} \leq T \leq T_{2}$, such that $T\left(\varphi_{n}\right)=y_{n}$ for all $n \in \mathbb{N} ; ;$

(b) If $J_{0} \subset \mathbb{N}$ is a finite subset, and $\left\{\lambda_{j} ; j \in J_{0}\right\} \subset \mathbb{R}$, then

$$
\sum_{i, j \in J_{0}} \lambda_{i} \lambda_{j} T_{1}\left(\varphi_{i+j+k}\right) \leq \sum_{i, j \in J_{0}} \lambda_{i} \lambda_{j} y_{i+j+k} \leq \sum_{i, j \in J_{0}} \lambda_{i} \lambda_{j} T_{2}\left(\varphi_{i+j+k}\right), k \in\{0,1\}
$$

In the scalar-valued case, we derive the following consequence:

Corollary 11. Let $v$ be a moment-determinate measure on $\mathbb{R}_{+}$. Assume that $h_{1}, h_{2}$ are two functions in $L_{v}^{\infty}\left(\mathbb{R}_{+}\right)$, such that $0 \leq h_{1} \leq h_{2}$ almost everywhere. Let $\left(y_{n}\right)_{n \geq 0}$ be a given sequence of real numbers. The following statements are equivalent:

(a) There exists $h \in L_{v}^{\infty}\left(\mathbb{R}_{+}\right)$, such that $h_{1} \leq h \leq h_{2} v$-almost everywhere, $\int_{\mathbb{R}_{+}} t^{j} h(t) d v=$ $y_{j}$ for all $j \in \mathbb{N} ; ;$

(b) If $J_{0} \subset \mathbb{N}$ is a finite subset, and $\left\{\lambda_{j} ; j \in J_{0}\right\} \subset \mathbb{R}$, then:

$$
\sum_{i, j \in J_{0}} \lambda_{i} \lambda_{j} \int_{\mathbb{R}_{+}} t^{i+j+k} h_{1}(t) d v \leq \sum_{i, j \in J_{0}} \lambda_{i} \lambda_{j} y_{i+j+k} \leq \sum_{i, j \in J_{0}} \lambda_{i} \lambda_{j} \int_{\mathbb{R}_{+}} t^{i+j+k} h_{2}(t) d v, k \in\{0,1\} .
$$

\subsection{On the Truncated Moment Problem}

The truncated moment problem is important in mathematics because it involves only a finite number of moments (of limited order), which are assumed to be known (or given, or measurable); therefore, it can be related to optimization problems [20] as well as to constructive methods for finding solutions to Markov moment problems [23,24]. For the existence of a polynomial solution see [28], where a symmetric positive definite matrix is naturally involved. Solution in $L^{\infty}$ spaces for the full moment problem as a weak limit of a sequence of solutions of truncated moment problems are discussed in [21]. The convergence holds in the weak topology of a $L^{\infty}$ space, with respect to the dual pair $\left(L^{1}, L^{\infty}\right)$. We start by recalling the truncated (reduced) Markov moment problems on a closed, bounded, or unbounded subset $F$ of $\mathbb{R}^{n}$, where $n \geq 1$ is an integer. We denote by $\mathbb{R}_{d}\left[t_{1}, \ldots t_{n}\right]$ the real vector subspace of all polynomial functions $\mathcal{P}$ of $n$ real variables, with real coefficients, generated by $t^{k}=t_{1}^{k_{1}} \cdots t_{n}^{k_{n}}, k_{i} \in\{0,1, \ldots, d\}, i=1, \ldots, n$, where $d \geq 1$ is a fixed integer. The dimension of this subspace is clearly equal to $N=(d+1)^{n}$. 
Given a finite set $\left\{m_{k}\right\} \quad 0 \leq k_{i} \leq d$, of real numbers, and a positive Borel measure $\mu$ on $i=1, \ldots, n$

$F$, with finite absolute moments $\int_{F}|t|^{k} d \mu<\infty$ for all $k=\left(k_{1}, \ldots, k_{n}\right) \in \mathbb{N}^{n}$ with $k_{i} \leq d$. $i=1, \ldots, n)$, one studies the existence and, eventual construction or approximation of a Lebesgue measurable real function $h \in L_{\mu}^{\infty}(F)$, satisfying the moment conditions

$$
\int_{F} t^{k} h(t) d \mu=m_{k}, k_{i} \leq d i=1, \ldots, n,
$$

and

$$
\mathbf{0} \leq h \leq b \mathbf{1}
$$

for some positive number $b$.

\subsection{Extension of Linear Operators, Markov Moment Problem, and Mazur-Orlicz Theorem}

The purpose of this section is to prove a result on an operator valued Markov moment problem whose solution is defined on a space of analytic functions, and an operator valued Mazur-Orlicz theorem involving the space of all real continuous functions defined on the spectrum of a self-adjoint operator as the domain space of the solution. Both these results follow as applications of a general theorem on extension for linear operators, with two constraints. They do not use any result on polynomial approximation. In the next theorem, $X$ will be a real vector space, $Y$ an order-complete vector lattice, $A, B \subseteq X$ convex subsets, $Q: A \rightarrow Y$ a concave operator, $P: B \rightarrow Y$ a convex operator, $M \subset X$ a vector subspace, and $T_{0}: M \rightarrow Y$ a linear operator. All vector spaces and linear operators are considered over the real field.

Theorem 13 (see [26]). Assume that $T_{0}(x) \geq Q(x) \forall x \in M \cap A, T_{0}(x) \leq P(x) \forall x \in M \cap B$. The following statements are equivalent:

(a) There exists a linear extension $T: X \rightarrow Y$ of the operator $T_{0}$ such that

$$
\left.T\right|_{A} \geq Q,\left.T\right|_{B} \leq P ;
$$

(b) There exists $P_{1}: A \rightarrow Y$, convex, and $Q_{1}: B \rightarrow Y$ concave operator such that for all

$$
\left(\rho, t, \lambda^{\prime}, a_{1}, a^{\prime}, b_{1}, b^{\prime}, v\right) \in[0,1]^{2} \times(0, \infty) \times A^{2} \times B^{2} \times M,
$$

the following implication holds:

$$
\begin{gathered}
(1-t) a_{1}-t b_{1}=v+\lambda^{\prime}\left((1-\rho) a^{\prime}-\rho b^{\prime}\right) \Rightarrow \\
(1-t) P_{1}\left(a_{1}\right)-t Q_{1}\left(b_{1}\right) \geq T_{0}(v)+\lambda^{\prime}\left((1-\rho) Q\left(a^{\prime}\right)-\rho P\left(b^{\prime}\right)\right) .
\end{gathered}
$$

It is worth noticing that the extension $T$ of Theorem 13 satisfies the following conditions: it is an extension of $T_{0}$, it is dominated by $P$ on $B$, and it dominates $Q$ on $A$. Here the convex subsets $A, B$ are arbitrary, with no restriction on the existence of relative interior points or on their position with respect to the subspace $M$. As a consequence of Theorem 13, one can prove the next result:

Theorem 14 (see [26]). Let $X$ be a locally convex space, $Y$ an order complete vector lattice with strong order unit $u_{0}$, and $S \subset X$ a vector subspace. Let $C \subset X$ be a convex subset with the following properties:

(a) There exists a neighborhood $D$ of the origin such that $(S+D) \cap C=\varnothing$ (that is, by definition, $C$ and $S$ are distanced);

(b) C is bounded. 
Then for any equicontinuous family of linear operators $\left\{f_{j}\right\}_{j \in J} \subset \mathcal{L}(S, Y)$ and for any $\widetilde{y} \in Y_{+} \backslash\{0\}$, there exists an equicontinuous family $\left\{T_{j}\right\}_{j \in J} \subset \mathcal{L}(X, Y)$ such that

$$
T_{j}(s)=f_{j}(s), s \in S, T_{j}(\psi) \geq \widetilde{y}, \psi \in C, j \in J .
$$

Moreover, if $D$ is a convex balanced neighbourhood of the origin such that

$$
f_{j}(D \cap S) \subset\left[-u_{0}, u_{0}\right],(S+D) \cap C=\varnothing
$$

and if $\alpha>0$ is such that $P_{D}(a) \leq \alpha \forall a \in C$ and $\alpha_{1}>0$ is large enough such that $\widetilde{y} \leq \alpha_{1} u_{0}$, then the following relations hold:

$$
T_{j}(x) \leq\left(1+\alpha+\alpha_{1}\right) P_{D}(x), x \in X, j \in J .
$$

We have denoted by $P_{D}$ the gauge (Minkowski functions) attached to $D$.

Let $\left(B_{k}\right)_{k \in \mathbb{N}^{n}}$ be a sequence of operators in $Y=Y(A)$ defined by (5) and $\|\widetilde{B}\| \in$ $Y_{+} \backslash\{0\}$. On the other hand, let $n \neq 0$ be a natural number and $X$ be the space of all absolutely convergent power series $h$ in the unit closed polydisk

$$
\bar{D}_{1}=\left\{z=\left(z_{1}, \ldots, z_{n}\right):\left|z_{i}\right| \leq 1, i \in\{1, \ldots, n\}\right\},
$$

with real coefficients. The norm on $X$ is defined by:

$$
\|h\|_{\infty}=\sup \left\{|h(z)|: z \in \bar{D}_{1}\right\} .
$$

We denote

$$
h_{k}(z)=z_{1}^{k_{1}} \cdots z_{n}^{k_{n}}, k=\left(k_{1}, \ldots, k_{n}\right) \in \mathbb{N}^{n}, z \in \bar{D}_{1},|k|=k_{1}+\cdots+k_{n} .
$$

Theorem 15 (see [29], Theorem 3.1, p. 178). Assume that $A_{1}, \ldots, A_{n}$ are elements of $Y(A)$ such that there exists a real number $M>0$, with the property

$$
\left|B_{k}\right| \leq M \frac{A_{1}^{2 k_{1}}}{k_{1} !} \cdots \frac{A_{n}^{2 k_{n}}}{k_{n} !}, \forall k \in \mathbb{N}^{n}, \sum_{p=1}^{n} A_{p}^{2} \leq I
$$

where $I: H \rightarrow H$ is the identity operator. Let $\left\{g_{k}\right\}_{k \in \mathbb{N}^{n}} \subset X$ be such that $1=g_{k \infty}=g_{k}(\mathbf{0}), \forall k \in$ $\mathbb{N}^{n}$. Then there exists a linear bounded operator $T \in B(X, Y)$ such that

$$
\begin{gathered}
T\left(h_{k}\right)=B_{k},|k| \geq 1, T\left(g_{k}\right) \geq \widetilde{B,} \forall k \in \mathbb{N}^{n}, \\
T(h) \leq\left(2+\|\widetilde{B}\| M^{-1} e^{-1}\right)\|h\|_{\infty} u_{0}, \forall h \in X, u_{0}=M e I
\end{gathered}
$$

In particular, the following evaluation holds: $\|T\| \leq 2 M e+\|\widetilde{B}\|$.

Proof. One applies Theorem 14, where $Y=Y(A)$ is defined in (5). The subspace generated by $\left\{h_{k}:|k| \geq 1\right\}$ stands for $S$ of Theorem 14 and the convex hull of the set of the functions $g_{k}, k \in \mathbb{N}^{n}$ stands for the set $C$. The following remark is essential

$$
|| s-\varphi \|_{\infty} \geq|s(0)-\varphi(\mathbf{0})|=|0-1|=1, \forall s \in S, \forall \varphi \in C .
$$

This proves that $(S+B(0,1)) \cap C=\varnothing$, so that $B(0,1)$ stands for $D$ and $\|\cdot\|=\|\cdot\|_{\infty}$ stands for $P_{D}$ of Theorem 14 . The operator $\widetilde{B}$ stands for $\widetilde{y}$. Now let

$$
\varphi=\sum_{j \in J_{0}} \beta_{j} h_{j} \in S \cap B(\mathbf{0}, 1),
$$


where $J_{0}$ is a finite subset of $\mathbb{N}^{n}$. The following relations hold:

$$
\left|\sum_{j \in J_{0}} \beta_{j} B_{j}\right| \leq \sum_{j \in J_{0}}\left|\beta_{j}\right|\left|B_{j}\right| \leq \varphi_{\infty} \sum_{j \in J_{0}} \frac{1}{r_{1}^{j_{1}} \cdots r_{n}^{j_{n}}}\left|B_{j}\right|,
$$

for any $0<r_{p}<1, p \in\{1, \ldots, n\}$, thanks to Cauchy inequalities. Passing to the limit with $r_{p} \uparrow 1, p \in\{1, \ldots, n\}$ and using the fact that $\varphi \in B(0,1)$, as well as the hypothesis in the statement, the preceding relation further yields

$$
\begin{gathered}
\left|\sum_{j \in J_{0}} \beta_{j} B_{j}\right| \leq \sum_{j \in J_{0}}\left|B_{j}\right| \leq M \sum_{j \in J_{0}} \frac{A_{1}^{2 j_{1}}}{j_{1} !} \cdots \frac{A_{n}^{2 j_{n}}}{j_{n} !} \leq M\left(\sum_{k_{1} \in \mathbb{N}} \frac{A_{1}^{2 k_{1}}}{k_{1} !}\right) \cdots\left(\sum_{k_{n} \in \mathbb{N}} \frac{A_{n}^{2 k_{n}}}{k_{n} !}\right) \\
=\operatorname{Mexp}\left(\sum_{p=1}^{n} A_{p}^{2}\right) \leq M \exp (I)=M e I=u_{0} .
\end{gathered}
$$

The conclusion is that denoting by $f: S \rightarrow Y$ the linear operator which satisfies the moment conditions $f\left(h_{k}\right)=B_{k}, k \in \mathbb{N}^{n},|k| \geq 1$, we have

$$
-M e I \leq f(s) \leq M e I=u_{0}, \forall s \in S \cap B(0,1) .
$$

On the other hand, the following relations hold:

$$
\widetilde{B} \leq\|\widetilde{B}\| I=\|\widetilde{B}\| M^{-1} e^{-1} u_{0}=\alpha_{1} u_{0},
$$

where $\alpha_{1}=\|\widetilde{B}\| M^{-1} e^{-1}$. The conditions on the norms of the functions $g_{k}, k \in \mathbb{N}^{n}$ lead to

$$
\|\varphi\| \leq 1, \forall \varphi \in C .
$$

So, the constant 1 stands for $\alpha$ from Theorem 14. Now all the conditions from the statement of Theorem 14 are accomplished. According to the latter theorem, there exists a linear mapping $T: X \rightarrow Y$, such that

$$
\begin{gathered}
T\left(h_{k}\right)=f\left(h_{k}\right)=B_{k}, k \in \mathbb{N}^{n},|k| \geq 1, T\left(g_{k}\right) \geq \widetilde{B}, \forall k \in \mathbb{N}^{n}, \\
T(h) \leq\left(2+\|\widetilde{B}\| M^{-1} e^{-1}\right)\|h\|_{\infty} M e I, \forall h \in X .
\end{gathered}
$$

From the last inequality, we derive

$$
|T(h)| \leq(2 M e+\|\widetilde{B}\|)\|h\|_{\infty} I, \forall h \in X .
$$

Since the norm on $Y$ is solid, we infer that

$$
\|T(h)\| \leq(2 M e+\|\widetilde{B}\|)\|h\|_{\infty}, \forall h \in X \Rightarrow\|T\| \leq 2 M e+\|\widetilde{B}\| .
$$

This concludes the proof.

We go on with the Mazur-Orlicz theorem in ordered vector space framework and one of its operator valued consequences.

Theorem 16 (see [25], Theorem 5). Let $X$ be a preordered vector space, $Y$ an order complete vector space, $\left\{x_{j}\right\}_{j \in J^{\prime}}\left\{y_{j}\right\}_{j \in J}$ families of elements in $X$, respectively in $Y$, and $P: X \rightarrow Y$ a sublinear operator. The following statements are equivalent:

(a) There exists a linear positive operator $T: X \rightarrow Y$ such that

$$
T\left(x_{j}\right) \geq y_{j}, j \in J, T(x) \leq P(x), x \in X ;
$$


(b) For any finite subset $J_{0} \subset J$ and any $\left\{\lambda_{j}\right\}_{j \in J_{0}} \subset \mathbb{R}_{+}$, the following implication holds true:

$$
\sum_{j \in J_{0}} \lambda_{j} x_{j} \leq x \in X \Rightarrow \sum_{j \in J_{0}} \lambda_{j} y_{j} \leq P(x) .
$$

Corollary 12. Let $A$ be a positive self-adjoint operator acting on a Hilbert space $H$, let $Y=Y(A)$ be defined by (5), and $X:=C(\sigma(A))$. Let $\left(B_{j}\right)_{j \in \mathbb{N}}$ be a sequence in $Y, \varphi_{j}(t)=t^{j}, j \in \mathbb{N}, t \in \sigma(A)$. The following statements are equivalent:

(a) There exists a linear bounded positive operator $T \in B_{+}(X, Y)$ such that

$$
T\left(\varphi_{j}\right) \geq B_{j}, j \in \mathbb{N}, T(\varphi) \leq \int_{\sigma(A)}|\varphi(t)| d E_{A}, \forall \varphi \in X,\|T\| \leq 1 ;
$$

(b) $\quad B_{j} \leq A^{j}, j \in \mathbb{N}$.

Proof. The spectrum $\sigma(A)$ is contained in $\mathbb{R}_{+}$, since $A$ is self-adjoint and positive. The implication $(\mathrm{a}) \Rightarrow(\mathrm{b})$ is obvious:

$$
B_{j} \leq T\left(\varphi_{j}\right) \leq \int_{\sigma(A)}\left|\varphi_{j}\right| d E_{A}=\int_{\sigma(A)} \varphi_{j} d E_{A}=A^{j}, j \in \mathbb{N} ;
$$

(we have used the positivity of the operator $A$ which leads to $\left|\varphi_{j}\right|=\varphi_{j}$ on $\sigma(U) \subset \mathbb{R}_{+}$). For the converse, one applies Theorem $16(\mathrm{~b}) \Rightarrow(\mathrm{a})$, where $J$ stands for $\mathbb{N}, x_{j}$ stands for $\varphi_{j}, \varphi_{j}(t)=t^{j}$ and $y_{j}$ stands for $B_{j}, \forall j \in \mathbb{N}$. Let $J_{0}$ and $\left\{\lambda_{j}\right\}_{j \in J_{0}}$ be as mentioned at point (b) of Theorem 16. The following implications hold

$$
\begin{gathered}
\sum_{j \in J_{0}} \lambda_{j} \varphi_{j} \leq \varphi \in X \Rightarrow \sum_{j \in J_{0}} \lambda_{j} \int_{\sigma(A)} \varphi_{j} d E_{A}=\sum_{j \in J_{0}} \lambda_{j} A^{j} \leq \\
\int_{\sigma(A)} \varphi d E_{A} \leq \int_{\sigma(A)}|\varphi| d E_{A}:=P(\varphi) \leq\|\varphi\| I
\end{gathered}
$$

The positivity of the spectral measure $d E_{A}$ has been used. On the other hand, hypothesis (b) and the fact that the scalars $\lambda_{j}$ are nonnegative and the preceding evaluation yield

$$
\lambda_{j} B_{j} \leq \lambda_{j} A^{j} \forall j \in J_{0} \Rightarrow \sum_{j \in J_{0}} \lambda_{j} B_{j} \leq \sum_{j \in J_{0}} \lambda_{j} A^{j} \leq P(\varphi),
$$

where $P(\varphi)$ was defined above. Thus the implication (b) of Theorem 16 is satisfied. Application of the latter theorem leads to the existence of a "feasible solution" $T$ having the property mentioned at point (a) of the present theorem. The last property is a consequence of the preceding one, using the fact that the norm on $Y$ is solid. Namely,

$$
\begin{gathered}
\pm T(\varphi)=T( \pm \varphi) \leq \int_{\sigma(A)}|\varphi| d E_{A}=P(\varphi) \Rightarrow \\
|T(\varphi)| \leq P(\varphi) \Rightarrow\|T(\varphi)\| \leq\|P(\varphi)\| \leq\|\varphi\| \cdot\|I\|=\|\varphi\|, \varphi \in X .
\end{gathered}
$$

Thus we have $\|T\| \leq 1$. This concludes the proof.

\section{Discussion}

This is an up to date paper on polynomial approximation and some of its applications, complete with recent applications of old general constrained extension theorems for linear operators. There are two such constraints (sandwich condition) on the linear extension. Old results have been reviewed without proofs, while results published in the last decade are accompanied by their proofs. There are some different points of view with respect to the papers $[27,28]$. For example, Section 3.4 was added and Section 3.1 was modified. It is worth 
noticing that solving the Markov moment problem is not the only direction for applications of polynomial approximation (see Theorem 11 proved above). On the other hand, old, basic results proved in [26], found recent applications in [22], to the characterization of the isotonic convex operators on convex cones, in terms of their subgradients. Having these all in mind, we hope that the results of Section 3 could find new applications and bring new ideas in this area of research.

Funding: This research received no external funding.

Institutional Review Board Statement: Not applicable.

Informed Consent Statement: Not applicable.

Data Availability Statement: Not applicable.

Acknowledgments: The author would like to thank the anonymous referees for their comments and suggestions, leading to the improvement of the presentation of this paper.

Conflicts of Interest: The author declares no conflict of interest.

\section{References}

1. Akhiezer, N.I. The Classical Moment Problem and Some Related Questions in Analysis; Oliver and Boyd: Edinburgh, UK, 1965.

2. Berg, C.; Christensen, J.P.R.; Ressel, P. Harmonic Analysis on Semigroups; Theory of Positive Definite and Related Functions; Springer: New York, NY, USA, 1984.

3. Schmüdgen, K. The Moment Problem. In Graduate Texts in Mathematics; Springer International Publishing AG: Cham, Switzerland, 2017; p. 277. [CrossRef]

4. Krein, M.G.; Nudelman, A.A. Markov Moment Problem and Extremal Problems; American Mathematical Society: Providence, RI, USA, 1977.

5. Cristescu, R. Ordered Vector Spaces and Linear Operators; Academiei: Bucharest, Romania; Abacus Press: Tunbridge Wells, UK, 1976.

6. Niculescu, C.; Popa, N. Elements of Theory of Banach Spaces; Academiei: Bucharest, Romania, 1981. (In Romanian)

7. Niculescu, C.P.; Persson, L.-E. Convex Functions and Their Applications, a Contemporary Approach, 2nd ed.; CMS Books in Mathematics; Springer: New York, NY, USA, 2018; Volume 23.

8. Kutateladze, S.S. Convex operators. Russ. Math. Surv. 1979, 34, 181-214. [CrossRef]

9. Haviland, E.K. On the momentum problem for distributions in more than one dimension. Am. J. Math. 1936, 58, 164-168. [CrossRef]

10. Berg, C.; Christensen, J.P.R.; Jensen, C.U. A remark on the multidimensional moment problem. Math. Ann. 1979, 243, 163-169. [CrossRef]

11. Berg, C.; Thill, M. Rotation invariant moment problems. Acta Math. 1991, 167, 207-227. [CrossRef]

12. Motzkin, T.S. The arithmetic-geometric inequality. In Inequalities, Proceedings of the a Symposium, Wright-Patterson Air Force Base, Dayton, OH, USA, 19-27 August 1965; Academic Press: New York, NY, USA, 1967; pp. 205-224.

13. Stoyanov, J.M.; Lin, G.D.; Kopanov, P. New checkable conditions for moment determinacy of probability distributions. SIAM Theory Probab. Appl. 2020, 65, 497-509. [CrossRef]

14. Cassier, G. Problèmes des moments sur un compact et $\mathbb{R}^{n}$ décomposition des polynômes à plusieurs variables (Moment problems on a compact subset of $\mathbb{R}^{n}$ and decomposition of polynomials of several variables). J. Funct. Anal. 1984, 58, 254-266. [CrossRef]

15. Schmüdgen, K. The K-moment problem for compact semi-algebraic sets. Math. Ann. 1991, 289, 203-206. [CrossRef]

16. Putinar, M. Positive polynomials on compact semi-algebraic sets. IU Math J. 1993, 42, 969-984. [CrossRef]

17. Vasilescu, F.H. Spectral measures and moment problems. In Spectral Analysis and Its Applications (Ion Colojoară Anniversary Volume); Theta: Bucharest, Romania, 2003; pp. 173-215.

18. Marshall, M. Polynomials non-negative on a strip. Proc. Am. Math. Soc. 2010, 138, 1559-1567. [CrossRef]

19. Fuglede, B. The multidimensional moment problem. Exp. Math. 1983, 1, 47-65.

20. Inverardi, P.L.N.; Tagliani, A. Stieltjies and Hamburger reduced moment problem when MaxEnt solution does not exist. Mathematics 2021, 9, 309. [CrossRef]

21. Stochel, J. Solving the truncated moment problem solves the full moment problem. Glasg. Math. J. 2001, 43, 335-341. [CrossRef]

22. Niculescu, C.P.; Olteanu, O. From the Hahn-Banach extension theorem to the isotonicity of convex functions and the majorization theory. Rev. Real Acad. Cienc. Exactas Fis. Nat. RACSAM 2020, 114, 171. [CrossRef]

23. Gosse, L.; Runborg, O. Resolution of the finite Markov moment problem. Comptes Rendus Acad. Sci. Paris 2005, 341, 775-780. [CrossRef]

24. Gosse, L.; Runborg, O. Existence, uniqueness, and a constructive solution algorithm for a class of finite Markov moment problems. SIAM J. Appl. Math. 2008, 68, 16181640. [CrossRef] 
25. Olteanu, O. Application de théorèmes de prolongement d'opérateurs linéaires au problème des moments e à une generalization d'un théorème de Mazur-Orlicz, (Applications of theorems on extension of linear operators to the moment problem and to a generalization of Mazur-Orlicz theorem). Comptes Rendus Acad. Sci. Paris 1991, 313, 739-742.

26. Olteanu, O. Théorèmes de prolongement d'opérateurs linéaires (Theorems of extension of linear operators). Rev. Roum. Math. Pures Appl. 1983, 28, 953-983.

27. Olteanu, O. Polynomial approximation on unbounded subsets, Markov moment problem and other applications. Mathematics 2020, 8, 1654. [CrossRef]

28. Olteanu, O. On Markov moment problem and related results. Symmetry 2021, 13, 986. [CrossRef]

29. Olteanu, O.; Mihăilă, J.M. Operator-valued Mazur-Orlicz and moment problems in spaces of analytic functions. UPB Sci. Bull. Ser. A 2017, 79, 175-184. 